\title{
End-to-end distribution function for dilute polymers
}

\author{
Sergio Caracciolo \\ Scuola Normale Superiore and INFN - Sezione di Pisa \\ I-56100 Pisa, ITALY \\ Internet: Sergio.Caracciolo@sns.it \\ Maria Serena Causo \\ John von Neumann-Institut für Computing (NIC) \\ Forschungszentrum Jülich \\ D-52425 Jülich, GERMANY \\ Internet: M.S.Causo@fz-juelich.de \\ Andrea Pelissetto \\ Dipartimento di Fisica and INFN - Sezione di Roma I \\ Università degli Studi di Roma "La Sapienza" \\ I-00185 Roma, ITALY \\ Internet: Andrea.Pelissetto@roma1.infn.it
}

September 5, 2018

\begin{abstract}
We study the end-to-end distribution function for dilute polymers. We present a computation to order $O\left(\epsilon^{2}\right), \epsilon=4-d$, and discuss in detail its asymptotic behaviour for small and large distances. The theoretical predictions are compared with Monte Carlo results, finding good agreement. We show that the McKenzie-Moore-des Cloizeaux phenomelogical ansatz provides a very precise approximation to the exact EEDF.
\end{abstract}




\section{Introduction}

The statistical properties of dilute polymers in good solvents have been the subject of extensive studies during the years [1 41. A significant understanding of the problem was reached when it was realized that long polymers could be modelled by chains with an excluded-volume interaction. This allowed the introduction of simplified theoretical models which could be analyzed more easily. From a theoretical point of view, an important step forward was made by de Gennes [5], who proved that the statistical properties of polymers could be obtained as the limit $N \rightarrow 0$ of the $N$-component $\phi^{4}$ theory, opening the field to the many methods that were developed at the time for the study of the critical behaviour of spin systems.

In this paper we consider the end-to-end distribution function (EEDF). During the last thirty years a lot of work has been devoted to the study of this quantity. Exact results were obtained in Refs. [6-11], and many numerical studies checked some of the theoretical predictions [12 20]. In particular, these works tried to understand which of the several phenomenological expressions [21 provided the best description of the numerical data. The proposal by Mazur [8] was clearly excluded [15], while the theoretically motivated form of McKenzie and Moore [9] and des Cloizeaux [3, 11] was confirmed to a good accuracy [17, 18, 20]. It is interesting to notice that a precise knowledge of the EEDF could be relevant in experimental studies. Indeed, as observed by des Cloizeaux and Jannink [3], the EEDF could be determined from scattering experiments with a dilute solution of polymers with marked endpoints. A measurement of the scattered intensity at large angles would determine the EEDF in the large-momentum region. This would provide an estimate of the critical exponent $\gamma$, which is otherwise inaccessible experimentally.

In this paper we reconsider the problem of the determination of the EEDF. We extend the $\epsilon$-expansion calculations of Ref. [22] to order $\epsilon^{2}$ and give perturbative expansions for the quantities that characterize the asymptotic behaviour for small and large distances. Using the $\epsilon$-expansion we can show that the phenomenological parametrization of [9, 11] is essentially exact for distances much larger than the correlation length - the expected discrepancy is of order 1-5\% in the region accessible to simulations - while in the opposite range the discrepancy should be (at most) of order $10 \%$. We also give theoretical expressions for several quantities derived from the EEDF. Using precise Monte Carlo estimates of the critical exponents, we derive accurate theoretical predictions for the asymptotic behaviour of the EEDF. The theoretical estimates are compared with numerical results obtained from a simulation of self-avoiding walks on a cubic lattice, finding good agreement.

The paper is organized as follows: in Sec. 2 we introduce our notations and definitions and review the exact results that are available for the EEDF. In Sec. 3 we report our computation of the EEDF to order $\epsilon^{2}$. Only the results are given, the technical details being presented in the Appendix. In Sec. 4 we obtain estimates for the asymptotic behaviour of the EEDF using the Laplace-de Gennes transform. Finally in Sec. 5 we discuss the numerical results. 


\section{Definitions}

We consider a monodisperse ensemble of polymers with $N$ monomers. If $\mathbf{r}$ is the vector joining the endpoints of the walk, we will be interested in computing the unnormalized distribution $c_{N}(\mathbf{r})$ [23] of the endpoint vector. We also introduce a normalized distribution

$$
P_{N}(\mathbf{r})=\frac{c_{N}(\mathbf{r})}{\sum_{r} c_{N}(\mathbf{r})},
$$

the mean squared end-to-end distance

$$
R_{e, N}^{2}=\sum_{r}|\mathbf{r}|^{2} P_{N}(\mathbf{r})
$$

and the related correlation length

$$
\xi_{N}^{2}=\frac{1}{2 d} R_{e, N}^{2}
$$

In the limit $N \rightarrow \infty,|\mathbf{r}| \rightarrow \infty$, with $|\mathbf{r}| N^{-\nu} \sim|\mathbf{r}| / \xi_{N}$ fixed, the function $P_{N}(\mathbf{r})$ has the scaling form [0], 9, 11]

$$
P_{N}(\mathbf{r}) \approx \frac{1}{\xi_{N}^{d}} f(\rho)\left[1+O\left(N^{-\Delta}\right)\right]
$$

where $\boldsymbol{\rho}=\mathbf{r} / \xi_{N}, \rho=|\boldsymbol{\rho}|, d$ is the space dimensionality, and $\Delta$ is a correction-toscaling exponent. By definition

$$
\begin{aligned}
& \int_{0}^{\infty} \rho^{d-1} d \rho f(\rho)=\frac{1}{S_{d}}, \\
& \int_{0}^{\infty} \rho^{d+1} d \rho f(\rho)=\frac{2 d}{S_{d}},
\end{aligned}
$$

where $S_{d}$ is the volume of the $d$-dimensional sphere

$$
S_{d}=\frac{2 \pi^{d / 2}}{\Gamma(d / 2)} .
$$

Several facts are known about $f(\rho)$. For large values of $\rho$ it behaves as [6, 0 , 9, 11]

$$
f(\rho) \approx f_{\infty} \rho^{\sigma} \exp \left(-D \rho^{\delta}\right)
$$

where $\sigma$ and $\delta$ are given by

$$
\begin{aligned}
\delta & =\frac{1}{1-\nu} \\
\sigma & =\frac{2 \nu d-2 \gamma+2-d}{2(1-\nu)}
\end{aligned}
$$

For $\rho \rightarrow 0$, we have 9, 11

$$
f(\rho) \approx f_{0}\left(\frac{\rho}{2}\right)^{\theta}
$$


where

$$
\theta=\frac{\gamma-1}{\nu}
$$

We can also consider the Fourier transform of $f(\rho)$,

$$
\tilde{f}(Q)=\int \frac{d^{d} \boldsymbol{\rho}}{(2 \pi)^{d}} e^{i \mathbf{Q} \cdot \boldsymbol{\rho}} f(\rho),
$$

which is the critical (large- $N$ ) limit of $\widetilde{c}_{N}(\mathbf{q}) / \widetilde{c}_{N}(\mathbf{0})$ with $\mathbf{Q} \equiv \mathbf{q} \xi_{N}$ fixed, $\widetilde{c}_{N}(\mathbf{q})$ being the Fourier transform of $c_{N}(\mathbf{r})$. For $Q \rightarrow 0, \tilde{f}(Q)$ has a regular expansion in powers of $Q^{2}$, while for $Q^{2} \rightarrow \infty$ it behaves as

$$
\tilde{f}(Q)=\tilde{f}_{\infty} Q^{-\theta-d}
$$

The constants $\tilde{f}_{\infty}$ and $f_{0}$ are related. Indeed

$$
f_{0}=\tilde{f}_{\infty}(4 \pi)^{-d / 2} \frac{\Gamma\left(-\frac{\theta}{2}\right)}{\Gamma\left(\frac{\theta+d}{2}\right)} .
$$

A phenomenological representation for the function $f(\rho)$ has been proposed by McKenzie and Moore [9] and des Cloizeaux [3]:

$$
f(\rho) \approx f_{\mathrm{ph}}(\rho)=f_{\mathrm{ph}} \rho^{\theta} \exp \left(-D_{\mathrm{ph}} \rho^{\delta}\right) .
$$

Here $\delta$ and $\theta$ are fixed by (2.9) and (2.12), while $f_{\mathrm{ph}}$ and $D_{\mathrm{ph}}$ are fixed by the normalization conditions (2.5) and (2.6):

$$
\begin{aligned}
D_{\mathrm{ph}} & =\left\{\frac{\Gamma[(1-\nu)(2+d+\theta)]}{2 d \Gamma[(1-\nu)(d+\theta)]}\right\}^{\frac{\delta}{2}}, \\
f_{\mathrm{ph}} & =\frac{\delta D_{\mathrm{ph}}^{(d+\theta)(1-\nu)}}{S_{d} \Gamma[(1-\nu)(d+\theta)]} .
\end{aligned}
$$

In two dimensions

$$
\begin{aligned}
D_{\mathrm{ph}} & =0.026339478 \ldots, \\
f_{\mathrm{ph}} & =0.046757638 \ldots,
\end{aligned}
$$

while in three dimensions, using the precise estimate $\nu=0.58758 \pm 0.00007$ [24] and our result [25] $\gamma=1.1575 \pm 0.0006$, we have

$$
\begin{aligned}
D_{\mathrm{ph}} & =0.14470 \pm 0.00014 \\
f_{\mathrm{ph}} & =0.015990 \pm 0.000008 \\
\theta & =0.2680 \pm 0.0011 \\
\delta & =2.4247 \pm 0.0004
\end{aligned}
$$

Notice that $f_{\mathrm{ph}}(\rho)$ cannot be exact since the exponents $\theta$ and $\sigma$ are different, but this is less crucial in $d=3$, since their numerical values are very similar. In order 
to improve the approximation, one can also take $\theta$ and $\delta$ as free parameters, and determine them by fitting numerical estimates of $f(\rho)$. However, once $\theta$ and $\delta$ are given, $D_{\mathrm{ph}}$ and $f_{\mathrm{ph}}$ are uniquely determined by (2.17) and (2.18). In the following we will indicate with "phenomenological representation" the function (2.16) with $\delta$ and $\theta$ fixed to their theoretical values.

For the purpose of computing $D$ and $\delta$ from Monte Carlo data, it is much easier to consider the "wall-to-wall" distribution function

$$
P_{N, w}(x)=\sum_{x_{2}, \ldots, x_{d}} P_{N}\left(x, x_{2}, \ldots, x_{d}\right),
$$

which represents the probability that the endpoint of the walk lies on a plane at a distance $x$ from the origin of the walk. In the large- $N$ limit, $P_{N, w}(x)$ has the scaling form

$$
P_{N, w}(x)=\frac{1}{\xi_{N}} f_{w}(\rho)\left(1+O\left(N^{-\Delta}\right)\right)
$$

where $\rho=x / \xi_{N}$. We will show in Sec. 田 that for large $\rho$ we have

$$
f_{w}(\rho) \approx f_{w, \infty} \rho^{\sigma_{w}} \exp \left(-D \rho^{\delta}\right)
$$

where $\delta$ is given by (2.9), $D$ is the same constant appearing in Eq. (2.8), and

$$
\sigma_{w}=\delta\left(\nu-\gamma+\frac{1}{2}\right)
$$

\section{Distribution function to order $O\left(\epsilon^{2}\right)$}

We will now derive the EEDF $f(\rho)$ using a continuum description and the standard $\epsilon$-expansion. We start from the Edwards' path integral [26] for the probability distribution function of the end-to-end distance $\mathbf{r}$ of a chain with contour length $N$ in $d$ space dimensions

$$
c_{N}(\mathbf{r})=\int_{\mathbf{x}(0)=\mathbf{0}}^{\mathbf{x}(N)=\mathbf{r}} D[\mathbf{x}] \exp \left\{-\frac{1}{4} \int_{0}^{N} d s\left(\frac{d \mathbf{x}(s)}{d s}\right)^{2}-\frac{w}{2} \int_{0}^{N} d s \int_{0}^{N} d t \delta[\mathbf{x}(s)-\mathbf{x}(t)]\right\} .
$$

Here $\mathbf{x}(s)$ is the position vector of the arc-length position $s$ of the chain, the integral over $D[\mathbf{x}]$ represents the summation over all possible configurations between the two ends of the chain, and $w$ is the unrenormalized strength of the excluded-volume interaction. We use adimensional units setting the Kuhn step length equal to $2 d$.

In this Section we report the computation of the EEDF $f(\rho)$ to order $\epsilon^{2}$, where as usual $\epsilon=4-d$, extending the results of Ref. [22]. The diagrams that need to be computed are reported in Fig. 11. We obtain:

- Graph (a)

$G_{a}\left(N q^{2}\right)=-\frac{1}{2} B\left(2-\frac{\epsilon}{2},-1+\frac{\epsilon}{2}\right)\left[\left(1-N q^{2}\right) e^{-N q^{2}}+\epsilon g_{1}\left(N q^{2}\right)+\epsilon^{2} g_{2}\left(N q^{2}\right)\right]+O\left(\epsilon^{2}\right)$ 
- Graph (b)

$$
\begin{aligned}
G_{b}\left(N q^{2}\right)= & \frac{1}{4}\left(-1+\frac{\epsilon}{2}\right) B\left(2-\frac{\epsilon}{2},-1+\frac{\epsilon}{2}\right)^{2} \times \\
& {\left[\left(1-N q^{2}\right) e^{-N q^{2}}+2 \epsilon g_{1}\left(N q^{2}\right)+4 \epsilon^{2} g_{2}\left(N q^{2}\right)\right]+O(\epsilon) ; }
\end{aligned}
$$

- Graph (c)

$$
\begin{aligned}
G_{c}\left(N q^{2}\right)= & \frac{1}{4} B\left(2-\frac{\epsilon}{2},-1+\frac{\epsilon}{2}\right)^{2} \times \\
& \left(1+\frac{N}{2} \frac{\partial}{\partial N}\right)\left[\left(1-N q^{2}\right) e^{-N q^{2}}+2 \epsilon g_{1}\left(N q^{2}\right)+4 \epsilon^{2} g_{2}\left(N q^{2}\right)\right]+O(\epsilon) ;
\end{aligned}
$$

- Graph (d)

$$
\begin{aligned}
G_{d}\left(N q^{2}\right)= & {\left[-\frac{3}{2 \epsilon^{2}}-\frac{3}{4 \epsilon}-\frac{3}{2}+\frac{\pi^{2}}{8}-\frac{r^{2}}{6}-\frac{3}{2} \log 2 r+\frac{3}{2} \log ^{2} 2 r\right] } \\
& \times\left[\left(1-N q^{2}\right) e^{-N q^{2}}+2 \epsilon g_{1}\left(N q^{2}\right)+4 \epsilon^{2} g_{2}\left(N q^{2}\right)\right] \\
& -\left(\frac{1}{8 \epsilon}+\frac{41}{96}+\frac{15}{16 r^{2}}-\frac{1}{4} \log 2 r-\frac{9}{8 r^{2}} \log 2 r\right)\left(N q^{2} e^{-N q^{2}}+\epsilon g_{3}\left(N q^{2}\right)\right) \\
& \quad+4 N_{2}\left(N q^{2}\right)+4 N_{3}\left(N q^{2}\right)+O(\epsilon)
\end{aligned}
$$

The functions $g_{1}\left(N q^{2}\right), g_{2}\left(N q^{2}\right), g_{3}\left(N q^{2}\right), N_{2}\left(N q^{2}\right)$, and $N_{3}\left(N q^{2}\right)$, are defined in the Appendix. The result for diagram (d) depends apparently on an arbitrary parameter $r$; note that the functions $N_{2}\left(N q^{2}\right)$ and $N_{3}\left(N q^{2}\right)$ depend also on $r$ in such a way to make the final result independent of $r$. In principle $r$ can be set to any value. We have kept it arbitrary, in order to have a check of the calculations: indeed the final results must not depend on $r$. At two loops we obtain for the unrenormalized $\widetilde{c}_{N}(\mathbf{q})$

$$
\widetilde{c}_{N}(\mathbf{q})=e^{-N q^{2}}+\widetilde{w} N^{\epsilon / 2} G_{a}\left(N q^{2}\right)+\widetilde{w}^{2} N^{\epsilon}\left[G_{b}\left(N q^{2}\right)+G_{c}\left(N q^{2}\right)+G_{d}\left(N q^{2}\right)\right]+O\left(\widetilde{w}^{3}\right),
$$

where $\widetilde{w}=w N_{d}$ and $N_{d}=2(4 \pi)^{-d / 2} / \Gamma(d / 2)$. The computation of the universal EEDF goes through several steps. First of all, we compute the correlation length $\xi_{N}$ using the definition (2.3) and then we express $N$ in terms of $\xi_{N}$. We obtain

$$
N=\xi_{N}^{2}\left[1+\alpha_{1} \widetilde{w} \xi_{N}^{\epsilon}+\alpha_{2} \widetilde{w}^{2} \xi_{N}^{2 \epsilon}+O\left(\widetilde{w}^{3}\right)\right]
$$

where

$$
\begin{aligned}
\alpha_{1}= & -\frac{1}{3 \epsilon}+\frac{1}{3}-\frac{\gamma_{E}}{6}+\frac{\epsilon}{6}\left(-1+\gamma_{E}-\frac{1}{4} \gamma_{E}^{2}-\frac{\pi^{2}}{24}\right), \\
\alpha_{2}= & \frac{5}{18 \epsilon^{2}}-\frac{35}{72 \epsilon}+\frac{5 \gamma_{E}}{18 \epsilon}-\frac{35}{72} \gamma_{E}+\frac{5}{36} \gamma_{E}^{2}-\frac{\pi^{2}}{54}-\frac{5}{48 r^{2}}+\frac{r^{2}}{54} \\
& +\frac{7}{36} \log 2 r+\frac{1}{8 r^{2}} \log 2 r-\frac{1}{6} \log ^{2} 2 r+\frac{4}{9}\left(N_{2}(0)+N_{2}^{\prime}(0)\right) .
\end{aligned}
$$


where $\gamma_{E} \approx 0.5772156649$ is the Euler's constant.

Substituting this expression into Eq. (3.6), $\widetilde{c}_{N}(\mathbf{q})$ becomes a series in $\widetilde{w} \xi_{N}^{\epsilon}$, with coefficients depending on the combination $\mathbf{q} \xi_{N} \equiv \mathbf{Q}$. One can then compute $\widetilde{f}(Q)=\widetilde{c}_{N}(\mathbf{q}) / \widetilde{c}_{N}(\mathbf{0})$. This quantity, once expressed in terms of $Q$, requires only a renormalization of the interaction strength $w$ in order to be finite. In the minimal subtraction scheme we have [3, 27]

$$
\widetilde{w}=w_{R}\left(1+\frac{4}{\epsilon} w_{R}+O\left(w_{R}^{2}\right)\right) .
$$

The expansion in terms of $w_{R}$ is finite. The critical theory is obtained replacing $w_{R}$ with its fixed-point value $w_{R}^{*}$,

$$
w_{R}^{*}=\frac{1}{4} \epsilon+\frac{21}{128} \epsilon^{2}+O\left(\epsilon^{3}\right) .
$$

The final result can be written in the form

$$
\widetilde{f}(Q)=e^{-Q^{2}}+\epsilon \widetilde{f}^{(1)}(Q)+\epsilon^{2} \widetilde{f}^{(2)}(Q)+O\left(\epsilon^{3}\right),
$$

where

$$
\begin{aligned}
\widetilde{f}^{(1)}(Q)= & \frac{1}{4} \widehat{g}_{1}\left(Q^{2}\right) \\
\widetilde{f}^{(2)}(Q)= & \left(\frac{13}{128}-\frac{\gamma_{E}}{32}\right) \widehat{g}_{1}\left(Q^{2}\right)+\frac{1}{16} \widehat{g}_{2}\left(Q^{2}\right)-\frac{1}{128} \widehat{g}_{3}\left(Q^{2}\right)+\frac{1}{4} \widehat{N}_{2}\left(Q^{2}\right)+\frac{1}{4} N_{3}\left(Q^{2}\right) \\
& +\left(\frac{1}{16}-\frac{\gamma_{E}}{32}\right) Q^{2} \widehat{g}_{1}^{\prime}\left(Q^{2}\right)+\frac{Q^{2}}{16} \widehat{g}_{2}^{\prime}\left(Q^{2}\right)-\frac{\pi^{2}}{768}\left(Q^{2}\right)^{2} e^{-Q^{2}}
\end{aligned}
$$

where, for any function $h\left(Q^{2}\right)$, we define

$$
\widehat{h}\left(Q^{2}\right)=h\left(Q^{2}\right)-e^{-Q^{2}} h(0)\left(1+Q^{2}\right)-e^{-Q^{2}} h^{\prime}(0) .
$$

From the results reported in the Appendix one can derive the asymptotic behaviour of $\tilde{f}(Q)$ in the limits $Q \rightarrow 0$ and $Q \rightarrow \infty$. For $Q \rightarrow 0$ we have

$$
\tilde{f}(Q)=\frac{1}{1+Q^{2}}\left\{1-\left(Q^{2}\right)^{2}\left[\frac{1}{2}+\frac{\epsilon}{32}+\left(\frac{3}{1024}+\frac{\pi^{2}}{768}+\frac{\widehat{b}_{2}}{2}\right) \epsilon^{2}+O\left(\epsilon^{3}\right)\right]+O\left(Q^{6}\right)\right\},
$$

where we have introduced $\widehat{b}_{2}$,

$$
\widehat{b}_{2}=-\frac{1}{1536} \int_{0}^{\infty} d x x^{4} K_{1}^{3}(x) \approx-0.000235007,
$$

where $K_{1}(x)$ is a Bessel function. For $Q^{2} \rightarrow \infty$ we have

$$
\widetilde{f}(Q) \approx \frac{1}{\left(Q^{2}\right)^{2}}\left[-\frac{\epsilon}{8}+\frac{\epsilon^{2}}{256}\left(11-12 \gamma_{E}\right)-\frac{3 \epsilon^{2}}{64} \log Q^{2}\right]+O\left(\epsilon^{3}\right),
$$


where terms of order $\log Q^{2} /\left(Q^{2}\right)^{3}$ have been discarded. The function $f(\rho)$ can be derived from $\tilde{f}(Q)$. We will be interested in its asymptotic behaviour for $\rho \rightarrow \infty$. Using the results of the Appendix, a lengthy computation gives

$$
\begin{aligned}
f(\rho) \approx & \frac{1}{16 \pi^{2}}+\frac{\epsilon}{64 \pi^{2}}\left(\log \frac{\rho}{2}+2 \log 4 \pi+\frac{1}{2} \gamma_{E}-1\right) \\
& +\frac{\epsilon^{2}}{512 \pi^{2}}\left[\log ^{2} \frac{\rho}{2}+\left(4 \log 4 \pi+\gamma_{E}+\frac{1}{4}\right) \log \frac{\rho}{2}+1+\frac{19}{24} \pi^{2}\right. \\
& \left.+\frac{1}{8} \gamma_{E}+\frac{1}{4} \gamma_{E}^{2}-4 \log 4 \pi+2 \gamma_{E} \log 4 \pi+4 \log ^{2} 4 \pi+4 A\right],
\end{aligned}
$$

where terms vanishing for $\rho \rightarrow 0$ have been neglected. The constant $A$ is defined in Eq. (A.43). Numerically $A \approx-1.30204$. The expressions (3.18) and (3.19) are compatible with the asymptotic behaviours (2.14) and (2.11) and give the following expansions for the constants $\tilde{f}_{\infty}$ and $f_{0}$ :

$$
\begin{aligned}
\tilde{f}_{\infty}= & -\frac{1}{8} \epsilon+\frac{1}{256} \epsilon^{2}\left(11-12 \gamma_{E}\right)+O\left(\epsilon^{3}\right) \\
f_{0}= & \frac{1}{16 \pi^{2}}+\frac{\epsilon}{64 \pi^{2}}\left(2 \log 4 \pi+\frac{1}{2} \gamma_{E}-1\right)+\frac{\epsilon^{2}}{512 \pi^{2}}\left(1+\frac{19}{24} \pi^{2}+\frac{1}{8} \gamma_{E}+\frac{1}{4} \gamma_{E}^{2}\right. \\
& \left.\quad-4 \log 4 \pi+2 \gamma_{E} \log 4 \pi+4 \log ^{2} 4 \pi+4 A\right)+O\left(\epsilon^{3}\right)
\end{aligned}
$$

A check of these results is provided by Eq. (2.15). It is easy to verify that the two previous expansions satisfy Eq. (2.15) to order $\epsilon$. Moreover, using Eq. (2.15), we can obtain [28] the contribution of order $\epsilon^{3}$ to $\tilde{f}_{\infty}$. Explicitly we have

$$
\left(-\frac{41}{4096}+\frac{21}{1024} \gamma_{E}-\frac{9}{1024} \gamma_{E}^{2}-\frac{9}{2048} \pi^{2}+\frac{33}{512} \zeta(3)-\frac{1}{64} A\right) \epsilon^{3}
$$

Since only three terms are available, it is difficult to obtain reliable estimates from these expansions. Setting simply $\epsilon=1$, we obtain

$$
\tilde{f}_{\infty} \approx-0.0557, \quad f_{0} \approx 0.0176
$$

We have also used the resummation method of Ref. [30], which takes into account the singularity structure of the Borel transform of the perturbative series. We obtain [31]

$$
\tilde{f}_{\infty} \approx-0.089 \pm 0.017, \quad f_{0} \approx 0.015 \pm 0.002
$$

The ratio $\tilde{f}_{\infty} / f_{0}$ can be determined precisely using Eq. (2.15) and the precise Monte Carlo determinations of the critical exponents:

$$
\frac{\tilde{f}_{\infty}}{f_{0}}=-4.883 \pm 0.017 .
$$

We can use this result to test the accuracy of the estimates (3.23) and (3.24). Using Eq. (3.23) we obtain $\tilde{f}_{\infty} / f_{0}=-3.16$, while Eq. (3.24) gives $f_{\infty} / f_{0}=-5.9 \pm 1.4$. The estimates have an error of approximately 20-30\%. It is reassuring that the errors obtained with the resummation method correctly describe the discrepancy. 


\section{Asymptotic behaviour via Laplace-de Gennes trans- form}

Improved estimates of the asymptotic behaviour of $f(\rho)$ can be obtained from the precise results that have been derived for $O(N)$ spin models, using the Laplace-de Gennes transform method [3, 5, 11]. We introduce in $d$ dimensions the two-point function

$$
G(\mathbf{r} ; \beta)=\sum_{N=0}^{\infty} e^{-\beta N} c_{N}(\mathbf{r})
$$

its Fourier transform

$$
\widetilde{G}(\mathbf{q} ; \beta)=\sum_{r} e^{i \mathbf{q} \cdot \mathbf{r}} G(\mathbf{r} ; \beta)
$$

and the correlation length

$$
\xi^{2}(\beta) \equiv \frac{1}{2 d} \frac{\sum_{r}|\mathbf{r}|^{2} G(\mathbf{r} ; \beta)}{\sum_{r} G(\mathbf{r} ; \beta)} .
$$

In the critical limit $G(\mathbf{r} ; \beta)$ and $\widetilde{G}(\mathbf{q} ; \beta)$ have the scaling form [32, 33]

$$
\frac{G(\mathbf{r} ; \beta)}{\sum_{\mathbf{r}} G(\mathbf{r} ; \beta)} \approx \frac{1}{\xi^{d}(\beta)} D(\rho), \quad \frac{\widetilde{G}(\mathbf{q} ; \beta)}{\widetilde{G}(\mathbf{0} ; \beta)} \approx \widetilde{D}(Q),
$$

where $\rho=r / \xi(\beta)$ and $Q \equiv|\mathbf{q}| \xi(\beta)$. The function $\widetilde{D}(Q)$ is the $d$-dimensional Fourier transform of $D(\rho)$.

Several facts are known about the function $\widetilde{D}(Q)$. For $Q \ll 1, \widetilde{D}(Q)$ has a regular expansion in terms of $Q^{2}$ as

$$
\widetilde{D}^{-1}(Q)=1+Q^{2}+\sum_{i=2}^{\infty} b_{n} Q^{2 n}
$$

The coefficients $b_{n}, n=2,3,4,5$ have been computed [34] in the $\epsilon$-expansion up to $O\left(\epsilon^{4}\right)$ and [35] in the fixed-dimension expansion in $d=3$ up to $O\left(g^{5}\right)$. It turns out they are extremely small and satisfy $b_{2} \gg b_{3} \gg b_{4} \ldots$ For $b_{2}$ the explicit formulae are

$$
\begin{aligned}
& b_{2}=-0.000235007 \epsilon^{2}\left[1+1.0632 \epsilon+O\left(\epsilon^{2}\right)\right] \\
& b_{2}=-0.00015432 g^{2}\left[1+0.0780213 g+0.0465896 g^{2}+O\left(g^{3}\right)\right] .
\end{aligned}
$$

Here $g$ is the renormalized four-point coupling [36 constant whose critical value 30, 37 40, in the normalization we use, is $g^{*} \approx 1.39$. The expansions for $b_{2}, b_{3}, \ldots$, can be resummed using the method of Ref. [30], obtaining [35] $b_{2} \approx(-3 \pm 1) \cdot 10^{-4}$. Analogously $b_{3} \sim 10^{-5}$. An exact-enumeration study confirmed these results and gave the bound $-3 \cdot 10^{-4} \lesssim b_{2} \lesssim 0$. In two dimensions estimates have been obtained from the analysis [41] of exact-enumeration expansions on the triangular, square and honeycomb lattices: $b_{2} \approx 0.00015(20),\left|b_{3}\right| \lesssim 3 \cdot 10^{-5}$. In the following we will not need the explicit values of the constants $b_{i}$. Indeed they are too small to give any 
numerically important effect. Thus, for $Q \rightarrow 0$, we can approximate $\widetilde{D}^{-1}(Q)$ with $1+Q^{2}$.

For $Q \gg 1$, the behaviour of $\widetilde{D}(Q)$ is predicted by a short-distance renormalizationgroup analysis 42, 43] and one has

$$
\widetilde{D}(Q)=\frac{D_{0}}{Q^{2-\eta}}+\frac{D_{1}}{Q^{2-\eta+(1-\alpha) / \nu}}+\frac{D_{2}}{Q^{2-\eta+1 / \nu}}+\ldots
$$

The exponents $\eta$ and $\alpha$ are related to the exponents $\gamma$ and $\nu$ by

$$
\begin{aligned}
\eta & =2-\frac{\gamma}{\nu} \\
\alpha & =2-d \nu
\end{aligned}
$$

The constants $D_{0}, D_{1}$ and $D_{2}$ have been computed in the $\epsilon$-expansion [33, 34]:

$$
\begin{aligned}
& D_{0}=1-0.0317391 \epsilon^{2}-0.0353978 \epsilon^{3}+O\left(\epsilon^{4}\right) \\
& D_{1}=\frac{1}{2}\left(1+\frac{5}{8} \epsilon-0.4822 \epsilon^{2}\right)+D_{13} \epsilon^{3}+O\left(\epsilon^{4}\right) \\
& D_{2}=-\frac{3}{2}\left(1+\frac{5}{24} \epsilon-0.17653 \epsilon^{2}\right)+D_{23} \epsilon^{3}+O\left(\epsilon^{4}\right) .
\end{aligned}
$$

The coefficients $D_{13}$ and $D_{23}$ are not known but satisfy the relation:

$$
D_{13}+D_{23}=0.05934
$$

Resumming the perturbative series, we obtain

$$
D_{0} \approx 0.97 \pm 0.02, \quad D_{1} \approx 0.71 \pm 0.04, \quad D_{2} \approx-1.70 \pm 0.06
$$

Using the longer series for $D_{1}+D_{2}$ we obtain $D_{1}+D_{2} \approx-0.97 \pm 0.02$. It has been remarked by Aharony and Fisher [33] that one can also rewrite

$$
D_{1}=\frac{1}{\alpha}\left(\gamma-1+O\left(\epsilon^{3}\right)\right)
$$

and thus, for $d=3$, using the known values of the critical exponents, we obtain a similar estimate $D_{1} \approx 0.67$.

Finally one can determine the large- $\rho$ behaviour of $D(\rho)$. For $\rho \rightarrow \infty$, using the notations of Ref. [35], we have

$$
D(\rho) \approx A^{*} \rho^{-(d-1) / 2} e^{-\sqrt{S_{M}^{*}} \rho}
$$

where $A^{*}$ and $S_{M}^{*}$ are non-perturbative constants. The constant $A^{*}$ can be related to the residue $S_{Z}^{*}$ of the propagator at the mass pole. It is given by

$$
S_{Z}^{*}=\left.\frac{1}{2} \frac{\partial^{2}}{\partial Q^{2}} D^{-1}(Q)\right|_{Q^{2}=-S_{M}^{*}}
$$


One obtains

$$
A^{*}=\frac{1}{2 S_{Z}^{*}}(2 \pi)^{(1-d) / 2}\left(S_{M}^{*}\right)^{(d-3) / 4} .
$$

The constants $S_{Z}^{*}$ and $S_{M}^{*}$ have been computed [35] in the $\epsilon$-expansion up to $O\left(\epsilon^{4}\right)$ and in the expansion in fixed dimension $d=3$ up to $O\left(g^{4}\right)$ :

$$
\begin{aligned}
S_{Z}^{*} & =1+0.000489100 \epsilon^{2}+0.000522425 \epsilon^{3}+O\left(\epsilon^{4}\right) \\
S_{M}^{*} & =1-0.000241274 \epsilon^{2}-0.000257303 \epsilon^{3}+O\left(\epsilon^{4}\right), \\
S_{Z}^{*} & =1+0.000335466 g^{2}+0.0000140322 g^{3}+O\left(g^{4}\right), \\
S_{M}^{*} & =1-0.000163057 g^{2}-0.0000088348 g^{3}+O\left(g^{4}\right) .
\end{aligned}
$$

It is evident from these expansions that both constants are one with very small corrections. Resumming the expansions, we obtain in three dimensions $S_{M}^{*} \approx 1-(3 \pm$ $1) \cdot 10^{-4}$ and $S_{Z}^{*} \approx 1+(5 \pm 1) \cdot 10^{-4}$. In two dimensions estimates have been obtained from the analysis of exact-enumeration expansions [41]: $S_{M}^{*} \approx 1+(1 \pm 2) \cdot 10^{-4}$, $S_{Z}^{*} \approx 1-(2 \pm 4) \cdot 10^{-4}$.

From the asymptotic behaviour of $D(\rho)$ we obtain corresponding predictions for $f(\rho)$, using the fact that [3, 11]

$$
c_{N}(\mathbf{r})=\frac{1}{2 \pi i} \int_{-i \infty}^{+i \infty} d \beta e^{\beta N} G(\mathbf{r} ; \beta) .
$$

We report here the results; the derivations can be found e.g. in [3, 11].

We begin by computing the large- $Q$ behaviour of $\widetilde{f}(Q)$. Using Eq. (4.8) we obtain the expression (2.14) with

$$
\tilde{f}_{\infty}=\frac{1}{\pi} \Gamma(\gamma) D_{1} \sin (\pi d \nu) \Gamma(d \nu)\left(\frac{\Gamma(\gamma)}{\Gamma(\gamma+2 \nu)}\right)^{(\theta+d) / 2}
$$

Using the numerical values of the exponents, we obtain in two dimensions

$$
\begin{aligned}
\theta & =\frac{11}{24}, \\
\frac{\tilde{f}_{\infty}}{D_{1}} & =-0.11062768 \ldots
\end{aligned}
$$

and in three dimensions

$$
\begin{aligned}
\theta & =0.2680 \pm 0.0011 \\
\frac{\tilde{f}_{\infty}}{D_{1}} & =-0.12393 \pm 0.00026 .
\end{aligned}
$$

Using Eq. (2.15), we obtain correspondingly

$$
\frac{f_{0}}{D_{1}}= \begin{cases}0.050548 \ldots \ldots & \text { for } d=2 \\ 0.02539 \pm 0.00014 & \text { for } d=3\end{cases}
$$


In two dimensions we do not have any prediction for $D_{1}$, but, on the basis of the $\epsilon$ expansion result, we expect $D_{1}$ to be of order 1 , so that a reasonable guess is $-0.05 \lesssim$ $\tilde{f}_{\infty} \lesssim-0.15$ and $0.02 \lesssim f_{0} \lesssim 0.07$. In three dimensions, using $D_{1} \approx 0.71 \pm 0.04$, we obtain $\tilde{f}_{\infty} \approx-0.088 \pm 0.006$ and $f_{0} \approx 0.018 \pm 0.001$. These estimates are in good agreement with the results obtained in Sec. 3 .

It is interesting to compare the small- $\rho$ behaviour of $f_{\mathrm{ph}}(\rho)$ with that of the exact function $f(\rho)$. Using Eq. (2.16), we obtain $f_{0, \mathrm{ph}} \approx f_{\mathrm{ph}} 2^{\theta} \approx 0.0193$, which does not differ significantly from the estimate of $f_{0}$ reported above. We can also compare the two predictions within the $\epsilon$-expansion. We have

$$
\frac{f_{0}}{f_{\mathrm{ph}} 2^{\theta}}=1+0.109663 \epsilon^{2}+O\left(\epsilon^{3}\right)
$$

which shows that the phenomenological approximation is essentially correct, with an expected discrepancy of order $10 \%$.

Using Eq. (4.17), one obtains the asymptotic behaviour (2.8) where $f_{\infty}$ and $D$ are given by

$$
\begin{aligned}
D & =\frac{1-\nu}{\nu}\left(\nu^{2} S_{M}^{*} \frac{\Gamma(\gamma)}{\Gamma(\gamma+2 \nu)}\right)^{\delta / 2} \\
f_{\infty} & =\frac{\left(S_{M}^{*}\right)^{(d-3) / 4}}{2 S_{Z}^{*}} \frac{\Gamma(\gamma)}{(2 \pi)^{d / 2}(1-\nu)^{1 / 2}}\left(\nu^{2} S_{M}^{*}\right)^{\delta(\nu(d+1)-2 \gamma+1) / 4}\left(\frac{\Gamma(\gamma)}{\Gamma(\gamma+2 \nu)}\right)^{\delta(2-2 \gamma+d) / 4}
\end{aligned}
$$

Using the values of $S_{M}^{*}$ and $S_{Z}^{*}$ we have reported before and the values of the exponents $\gamma$ and $\nu$ we get in two dimensions:

$$
\begin{aligned}
\delta & =4, \\
\sigma & =\frac{5}{8}, \\
D & =0.02771 \pm 0.00001, \\
f_{\infty} & =0.04273 \pm 0.00002 .
\end{aligned}
$$

In three dimensions, using $\nu=0.58758 \pm 0.00007$ [24] and our result [25] $\gamma=1.1575 \pm$ 0.0006 , we obtain

$$
\begin{aligned}
\delta & =2.4247 \pm 0.0004 \\
\sigma & =0.255 \pm 0.002, \\
D & =0.1434 \pm 0.0002, \\
f_{\infty} & =0.01581 \pm 0.00002 .
\end{aligned}
$$

Notice that the estimates of $D$ and $f_{\infty}$ would not have significantly changed, had we used the Gaussian values $S_{M}^{*}=S_{Z}^{*}=1$. In three dimensions the error is dominated by the error on $\gamma$ and $\nu$.

We can also use Eqs. (4.32) and (4.33) to derive $\epsilon$ - and $g$-expansions for $D$ and $f_{\infty}$. We obtain in $4-\epsilon$ dimensions

$$
\begin{aligned}
D & =\frac{1}{4}-0.0877837 \epsilon-0.0327168 \epsilon^{2}+0.0394476 \epsilon^{3}+O\left(\epsilon^{4}\right) \\
f_{\infty} & =\frac{1}{16 \pi^{2}}+0.00579036 \epsilon+0.00223513 \epsilon^{2}+0.00152100 \epsilon^{3}+O\left(\epsilon^{4}\right) ;
\end{aligned}
$$


in fixed dimension $d=3$ we get

$$
\begin{aligned}
D & =\frac{1}{4}-0.0877837 g+0.0153578 g^{2}-0.0104654 g^{3}+O\left(g^{4}\right) \\
f_{\infty} & =\frac{1}{(2 \pi)^{3 / 2}}-0.00534423 g+0.000981669 g^{2}-0.000734351 g^{3}+O\left(g^{4}\right) .
\end{aligned}
$$

Resumming the expansions using the method of Ref. [30], we obtain in the two cases: the $\epsilon$-expansion gives $D=0.1461 \pm 0.0040, f_{\infty}=0.0138 \pm 0.006$, while the $g$-expansion gives $D=0.1445 \pm 0.0015, f_{\infty}=0.01602 \pm 0.00010$. These results are less accurate than the previous ones, that were obtained using the precise Monte Carlo estimates of the critical exponents. Note that the estimate of $f_{\infty}$ obtained using the $\epsilon$-expansion is not compatible, with the quoted error bars, with the estimate (4.41). This is not surprising since in the expansion (4.43) all coefficients have the same sign: therefore, a Borel resummation based on the large-order behaviour of the coefficients (that predicts coefficients alternating in sign) is not expected to work well.

It is interesting to compare the estimates (4.40) and (4.41) with the phenomenological approximation (2.21) and (2.22). It is remarkable that the discrepancy is so tiny, precisely of $0.8 \%$ for $D$ and of $1.1 \%$ for $f_{\infty}$. Also in two dimensions the phenomenological approximation works reasonably well: the discrepancy is of $5 \%$ for $D$ and of $9 \%$ for $f_{\infty}$. This nice agreement can be understood within the $\epsilon$-expansion. Indeed

$$
\begin{aligned}
\frac{D}{D_{\mathrm{ph}}} & =1-0.012109 \epsilon^{2}+0.0039898 \epsilon^{3}+O\left(\epsilon^{4}\right), \\
\frac{f_{\infty}}{f_{\mathrm{ph}}} & =1-0.0083917 \epsilon^{2}-0.0065622 \epsilon^{3}+O\left(\epsilon^{4}\right) .
\end{aligned}
$$

The $\epsilon$-expansions of $D$ and $f_{\infty}$ and of their phenomenological approximations $D_{\mathrm{ph}}$ and $f_{\mathrm{ph}}$ differ by terms that are very small. Setting $\epsilon=1$, one finds an expected difference of order $1 \%$, in agreement with the estimates above.

Notice that also the exponent $\sigma$ does not differ significantly from $\theta$ in three dimensions. This explains the success of the phenomenological approximation (2.16) for $\rho \rightarrow \infty$. Indeed, in this limit, we have

$$
\frac{f_{\mathrm{ph}}(\rho)}{f(\rho)} \approx \frac{f_{\mathrm{ph}}}{f_{\infty}} \rho^{\theta-\sigma} \exp \left[-\left(D_{\mathrm{ph}}-D\right) \rho^{\delta}\right] \approx 1.011 \rho^{0.013} \exp \left(-0.0013 \rho^{\delta}\right),
$$

so that

$$
\frac{f(\rho)-f_{\mathrm{ph}}(\rho)}{f(\rho)} \approx \begin{cases}1 \% & \text { for } \rho=2 \\ 3 \% & \text { for } \rho=5 \\ 26 \% & \text { for } \rho=10\end{cases}
$$

However $f(5) \approx 2 \cdot 10^{-5}$, and $f(10) \approx 8 \cdot 10^{-19}$, so that in practice $f(\rho)$ can be sampled up to $\rho \approx 5-6$. Therefore, in the region accessible to numerical simulations, $f_{\mathrm{ph}}(\rho)$ provides an accurate description of the large- $\rho$ behaviour of the EEDF. 
Finally, we can use (4.5) to get predictions for the moments of $f(\rho)$. It is simple to show that the invariant ratios

$$
M_{2 k}=\frac{\sum_{r}|\mathbf{r}|^{2 k} P_{N}(\mathbf{r})}{\left(\sum_{r}|\mathbf{r}|^{2} P_{N}(\mathbf{r})\right)^{k}}
$$

approach, for $N \rightarrow \infty$, universal constants $M_{2 k}^{*}$ given by

$$
M_{2 k}^{*}=\frac{\Gamma(\gamma+2 \nu)^{k}}{\Gamma(\gamma+2 k \nu) \Gamma(\gamma)^{k-1}}\left[1-b_{2}(k-1)\right] k ! \prod_{j=0}^{k-1}\left(1+\frac{2 j}{d}\right),
$$

where, we have neglected all $b_{n}$ with $n \geq 3$ and all powers of $b_{2}$. Notice that Eq. (4.51) is exact for $k=2$.

Again, we can compare the exact expression (4.51) with the prediction obtained by using the phenomenological representation (2.16):

$$
M_{\mathrm{ph}, 2 k}^{*}=\Gamma\left(\frac{2 k+\theta+d}{\delta}\right) \Gamma\left(\frac{\theta+d}{\delta}\right)^{k-1} \Gamma\left(\frac{2+\theta+d}{\delta}\right)^{-k} .
$$

Numerically we have in three dimensions:

$$
\begin{aligned}
M_{4}^{*}=1.51397(79) & M_{\mathrm{ph}, 4}^{*}=1.50876(23), \\
M_{6}^{*}=3.018(4) & M_{\mathrm{ph}, 6}^{*}=2.993(1), \\
M_{8}^{*}=7.392(15) & M_{\mathrm{ph}, 8}^{*}=7.292(5), \\
M_{10}^{*}=21.35(6) & M_{\mathrm{ph}, 10}^{*}=20.94(2) .
\end{aligned}
$$

The phenomenological predictions are definitely not exact, but they show very small differences with respect to the exact ones. Since the moments define uniquely the distribution function, this implies that Eq. (2.16) is not only a good approximation in the regions of large and small values of $\rho$, but that it also provides a good overall parametrization of the EEDF.

We can also consider the "wall-to-wall" distribution $P_{N, w}(x)$. It is easy to see that for large $\rho$ we have Eq. (2.27) with

$$
\begin{aligned}
\sigma_{w} & =\delta\left(\nu-\gamma+\frac{1}{2}\right) \\
f_{w, \infty} & =f_{\infty}(2 \pi \nu)^{(d-1) / 2}\left(\frac{\nu^{2} S_{M}^{*} \Gamma(\gamma)}{\Gamma(\gamma+2 \nu)}\right)^{\delta(1-d) / 4} .
\end{aligned}
$$

In two dimensions we obtain

$$
\begin{aligned}
\sigma_{w} & =-\frac{3}{8} \\
f_{w, \infty} & =0.32167 \pm 0.00017
\end{aligned}
$$

and in three dimensions

$$
\begin{aligned}
\sigma_{w} & =-0.1695 \pm 0.0016 \\
f_{w, \infty} & =0.2855 \pm 0.0004
\end{aligned}
$$




\section{Monte Carlo study of the distribution function}

The EEDF has been extensively studied numerically in three dimensions. The Monte Carlo work essentially focused on the exponents and verified that the data could be well described by the phenomenological expression (2.16). Baumgärtner [14 computed the exponent $\delta$ obtaining

$$
\delta=2.44 \pm 0.05
$$

in very good agreement with the theoretical result (4.38). The exponent $\theta$ appearing in the phenomenological expression (2.16) was computed by several groups, obtaining

$$
\theta= \begin{cases}0.270 \pm 0.006 & \text { Ref. [14], } \\ 0.27 & \text { Ref. [17], } \\ 0.262 \pm 0.013 & \text { Ref. [18], } \\ 0.224 \pm 0.006 & \text { Ref. [19]. }\end{cases}
$$

All estimates but the last one do not differ sensibly from our theoretical results for $\sigma$ and $\theta$, and as expected, they lie between these two estimates. The estimate of Ref. [19] is instead too low; probably, the numerical data are affected by large corrections to scaling.

In this Section we want to extend these numerical analyses, checking the renormalization-group predictions presented in the previous Sections. We will not use the phenomenological expression (2.16), but we will compute the exponents $\delta, \sigma$, and $\theta$ studying the large- $\rho$ and small- $\rho$ behaviour of the EEDF. At the same time we will be able to compute the constants $f_{0}, f_{\infty}$ and $D$ and to compare them with the theoretical predictions.

In order to compute the EEDF, we have generated $N$-step self-avoiding walks on a three dimensional cubic lattice, using the pivot algorithm [44 47. Since in three dimensions corrections to scaling are particularly strong, we generated long walks with $500 \leq N \leq 32000$.

First of all, we have checked the prediction (4.51) for the invariant ratios $M_{2 k}$, for $k=2,3,4,5$. In Table 1 we report the Monte Carlo estimates of $M_{2 k}$ for various values of $N$. We have performed the extrapolation to $N \rightarrow \infty$ using

$$
M_{2 k}=M_{2 k}^{*}+B_{2 k}\left(\frac{8000}{N}\right)^{\Delta} .
$$

The final estimates are compatible with the less precise results of Ref. [15] and are in very good agreement with the theoretical predictions. The corrections to scaling appear to be quite important: we estimate $\Delta \approx 0.55 \pm 0.08$, in agreement with the renormalization-group and Monte Carlo predictions [48]. Note that, at our level of precision, the phenomenological predictions (4.52) are not consistent with the numerical data.

It is interesting to observe that we can use Eq. (4.51) to obtain independent estimates of the critical exponents. For instance, using $b_{2}=-(3 \pm 1) \cdot 10^{-4}$ [35], $\nu=0.58758 \pm 0.00007$ [24], and the Monte Carlo result for $M_{4}^{*}$, we obtain

$$
\gamma=1.1576 \pm 0.0013
$$


which is in perfect agreement with the more precise estimate of Ref. [25]. It should be noticed that most of the error on $\gamma$ is due to the error on $M_{4}^{*}$, and, indeed, with the existing estimates of $\nu$ and $b_{2}$, it would be possible to obtain with this method an estimate of $\gamma$ as precise as that given in Ref. [25]. By considering $M_{4}^{*}$ and $M_{6}^{*}$ one can try to estimate $\nu$ and $\gamma$ simultaneously. One obtains $\nu=0.588(6), \gamma=1.159(24)$. Finally, using the estimates of $\gamma$ and $\nu$ and our Monte Carlo result for $M_{4}^{*}$, we obtain a bound on $b_{2}$. We get $\left|b_{2}\right|<1.4 \cdot 10^{-3}$.

In addition to $M_{2 k}$, one can consider the invariant ratios

$$
K_{2 k}=\frac{\sum_{r}\left(x^{2 k}+y^{2 k}+z^{2 k}\right) P_{N}(\mathbf{r})}{\left[\sum_{r}\left(x^{2}+y^{2}+z^{2}\right) P_{N}(\mathbf{r})\right]^{k}} .
$$

The rotational invariance of the critical limit gives

$$
R_{2 k}=\frac{K_{2 k}}{M_{2 k}} \rightarrow \frac{3}{2 k+1}
$$

In Table 2 we report the estimates of the ratios $R_{2 k}$ for $k=2,3,4,5$ and several values of $N$. Notice that, in this case, corrections to scaling are practically absent. This is in agreement with the analysis of Ref. [35] which showed that quantities like $R_{2 k}$ have corrections of the form $N^{-\tau}$ with $\tau \approx 2 \nu$. Thus, they are much smaller than the standard corrections which behave as $N^{-\Delta} \sim N^{-0.5}$.

Let us now consider the EEDF itself. The computation of $P_{N}(x)$ from the Monte Carlo data is straightforward, but it is less clear how to estimate the error bars. If the walks are generated independently, and $\hat{p}(x)$ is the estimate of $P_{N}(x)$, the error is

$$
\left[\frac{1}{N_{M C}} \hat{p}(x)(1-\hat{p}(x))\right]^{\frac{1}{2}},
$$

where $N_{M C}$ is the number of Monte Carlo iterations. In our case, however, the walks are not generated independently. Therefore, one should take into account the autocorrelation time of the algorithm and the fact that estimates at different values of $x$ are correlated. In practice it is not feasible to take into account all these effects. We have simply observed that since global observables decorrelate after a few accepted pivot moves, a reasonable estimate of the errors can be obtained by replacing in Eq. (5.7) $N_{M C}$ with $f_{N} N_{M C}$, where $f_{N}$ is the acceptance fraction of the algorithm. Correlations between different points are neglected.

The functions $f(\rho)$ and $f_{w}(\rho)$ are reported in Figs. 2 and 3. The data fall on a single curve as expected: within the accuracy of the plot, no corrections to scaling are visible, but, as we shall discuss later, corrections are present if one looks at the data in more detail.

Let us now study the asymptotic behaviour of the EEDF. We will begin by considering the wall-to-wall EEDF $f_{w}(\rho)$. In order to study its large- $\rho$ behaviour, we have performed two different sets of fits:

$$
\begin{aligned}
\log \left(f_{w}(\rho)\right) & =\log f_{w, \infty}-D \rho^{\delta}, \\
\log \left(\rho^{0.169} f_{w}(\rho)\right) & =\log f_{w, \infty}-D \rho^{\delta} .
\end{aligned}
$$


In the first fit we have neglected the power term $\rho^{\sigma_{w}}$ that appears in the asymptotic behaviour of $f_{w}(\rho)$, in the second one we have taken this term into account using the theoretical prediction for $\sigma_{w}$, Eq. (4.61). For $\rho \rightarrow \infty$, both fits should give the correct result for $D$ and $\delta$, while only the second one gives an estimate of $f_{w, \infty}$. There are two types of systematic errors in these fits. First, there are scaling corrections: the scaling curve is obtained only in the limit $N \rightarrow \infty$. Secondly, there are non-asymptotic corrections: Eqs. (5.8) and (5.9) are valid only asymptotically for $\rho \rightarrow \infty$. In order to detect scaling corrections, we have performed fits at fixed values of $N$. Then, we have compared the results, looking for systematic variations of the estimates with the length of the walk. The final result is obtained including in the fit only walks with $N \geq N_{\min }$, where $N_{\min }$ is chosen so that the estimates for all $N \geq N_{\min }$ are independent of $N$ within error bars. A similar strategy has been used to detect nonasymptotic effects: we have performed several fits using in each case only data with $\rho \geq \rho_{\min }$. Looking at the variation of the estimates with $\rho_{\min }$, we can estimate the non-asymptotic corrections. The results of the fits for fixed values of $N$ and for three different values of $\rho_{\text {min }}$ are reported in Table 3. Apparently they do not show any systematic dependence on $N$, except perhaps $N=500$ and $N=1000$ : indeed the estimate of $\delta$ for $N=500,1000$ are slightly higher than the estimates obtained for larger values of $N$, while the estimates of $D$ are slightly smaller. One may suspect that these results are affected by scaling corrections of size comparable with the statistical error. For this reason, our final estimates are obtained using all data with $N \geq 2000$ only. We obtain from the first fit

$$
\begin{aligned}
& \delta= \begin{cases}2.413 \pm 0.006 & \rho_{\min }=3.0 \\
2.420 \pm 0.014 & \rho_{\min }=3.5\end{cases} \\
& D= \begin{cases}0.150 \pm 0.002 & \rho_{\min }=3.0 \\
0.148 \pm 0.004 & \rho_{\min }=3.5\end{cases}
\end{aligned}
$$

The second fit gives

$$
\begin{aligned}
& \delta= \begin{cases}2.458 \pm 0.006 & \rho_{\min }=3.0 \\
2.455 \pm 0.013 & \rho_{\min }=3.5\end{cases} \\
& D= \begin{cases}0.136 \pm 0.002 & \rho_{\min }=3.0 \\
0.137 \pm 0.004 & \rho_{\min }=3.5\end{cases}
\end{aligned}
$$

These results are in good agreement with the theoretical estimates reported above, cf. Eqs. (4.38) and (4.40). Indeed, increasing $\rho_{\text {min }}$, we observe the expected convergence to the theoretical results. The second fit gives also an estimate of $f_{w, \infty}$ : we find

$$
f_{w, \infty}= \begin{cases}0.274 \pm 0.002 & \rho_{\min }=3.0 \\ 0.276 \pm 0.006 & \rho_{\min }=3.5\end{cases}
$$

which converges to the estimate (4.62) for large values of $\rho_{\min }$.

In order to estimate $\sigma_{w}$ and $f_{w, \infty}$, we have performed fits of the form

$$
\log \left[f_{w}(\rho) \exp \left(D \rho^{\delta}\right)\right]=\log f_{w, \infty}+\sigma_{w} \log \rho,
$$

using the theoretical estimates of $D$ and $\delta$, for various values of $\rho \geq \rho_{\min }$. These fits are extremely unstable. Indeed the EEDF drops rapidly to zero (see Fig. 3) so that 
the fit uses data in a small interval in which $\log \rho$ does not vary significantly. Results with reasonable errors can be obtained only for $\rho_{\min } \lesssim 3$, and thus we have analyzed the data with $\rho_{\min }=2,2.5$ and 3 . The results for fixed values of $N$ are reported in Table 4 . Looking at the table, one immediately sees that there are strong nonasymptotic corrections. Clearly the asymptotic behaviour sets in only for very large values of $\rho$. Looking at the data with $\rho_{\min }=3.0$, one sees that the estimates show a systematic trend as $N$ increases. If we analyze together all data with $N \geq N_{\min }$ we have $\left(\rho_{\min }=3.0\right)$

$$
\begin{aligned}
& \sigma_{w}=\left\{\begin{array}{lc}
-0.184 \pm 0.004 & N_{\min }=1000, \\
-0.173 \pm 0.005 & N_{\min }=2000 \\
-0.169 \pm 0.005 & N_{\min }=4000, \\
-0.157 \pm 0.006 & N_{\min }=8000,
\end{array}\right. \\
& f_{w, \infty}= \begin{cases}0.289 \pm 0.002 & N_{\min }=1000 \\
0.284 \pm 0.002 & N_{\min }=2000, \\
0.282 \pm 0.002 & N_{\min }=4000, \\
0.278 \pm 0.002 & N_{\min }=8000 .\end{cases}
\end{aligned}
$$

The presence of confluent corrections and of non-asymptotic terms of opposite sign makes difficult to evaluate reliably $\sigma_{w}$ and $f_{w, \infty}$ : clearly large values of $N$ are needed to see the scaling regime and large values of $\rho$ are required to observe the correct asymptotic behaviour. From the results of the fits above we can conclude that there is a reasonable agreement between the theoretical estimates and the numerical results although a precise quantitative check is difficult.

Let us now consider the distribution function $f(\rho)$. In order to determine this function, we have computed the probability $P_{n}(\mathbf{r})$ from the Monte Carlo data. A graph of this quantity as a function of $r^{2}$ shows strong oscillations due to the underlying lattice structure. In order to reduce these effects, we have used a procedure analogous to that used in Ref. [17]. Given a number $N_{s h}$, we define $r_{n}^{2}=n N_{s h}$ and an averaged distribution function

$$
P_{N}^{a v}\left(r_{n}\right)=\frac{1}{N_{n}} \sum_{\mathbf{r}: r_{n-1}^{2}<r^{2} \leq r_{n}^{2}} P_{N}(\mathbf{r}),
$$

where $N_{n}$ is the number of lattice points in the shell $r_{n-1}^{2}<r^{2} \leq r_{n}^{2}$. For $N_{s h}$ fixed, in the scaling limit $|\mathbf{r}| \rightarrow \infty, N \rightarrow \infty$, with $\rho$ fixed, $P_{N}^{a v}(r)$ converges to $f(\rho)$, so that one can use the distribution (5.18) in order to compute the EEDF. The advantage is that lattice oscillations disappear in the averaging procedure. Of course, one should always check that the results do not depend on $N_{s h}$. As expected, as long as the number of points falling in each shell is sufficiently large and $\sqrt{N_{s h}} \ll \xi_{N}$, the final estimates are not sensitive to $N_{s h}$.

We have closely repeated the analysis performed for the wall-to-wall EEDF. The final results are in reasonable agreement with the theoretical predictions. First, we have performed two different sets of fits in order to determine $D$ and $\delta$. As before, we consider

$$
\begin{aligned}
\log (f(\rho)) & =\log f_{\infty}-D \rho^{\delta} \\
\log \left(\rho^{-0.255} f(\rho)\right) & =\log f_{\infty}-D \rho^{\delta}
\end{aligned}
$$


for various values of $\rho_{\min }$. The second fit keeps into account the presence of $\rho^{\sigma}$ using the theoretical prediction for $\sigma$, Eq. (4.39). The results of the fits for fixed values of $N$ and different $N_{s h}$ are reported in Table 5. No significant dependence on $N_{\min }$ and $\rho_{\min }$ is visible in these results. Considering all data with $N \geq 1000$, and using for each $N$ the largest $N_{s h}$ appearing in Table 5 , we obtain from the first fit

$$
\begin{aligned}
\delta & =\left\{\begin{array}{lc}
2.504 \pm 0.002 & \rho_{\min }=3.0 \\
2.481 \pm 0.004 & \rho_{\min }=3.5
\end{array}\right. \\
D & = \begin{cases}0.1222 \pm 0.0004 & \rho_{\min }=3.0 \\
0.1277 \pm 0.0008 & \rho_{\min }=3.5\end{cases}
\end{aligned}
$$

while the second one gives

$$
\begin{aligned}
\delta & = \begin{cases}2.444 \pm 0.002 & \rho_{\min }=3.0 \\
2.441 \pm 0.004 & \rho_{\min }=3.5\end{cases} \\
D & = \begin{cases}0.1397 \pm 0.0005 & \rho_{\min }=3.0 \\
0.1406 \pm 0.0009 & \rho_{\min }=3.5\end{cases}
\end{aligned}
$$

Fit (5.19) gives estimates that show strong scaling corrections, clearly due to the neglected power term. The asymptotic values are difficult to estimate from this fit. In any case, we should observe that the estimates have the correct trend towards the expected results. The second fit is more stable. The estimates are in much better agreement with the theoretical results, although larger values of $\rho_{\min }$ are necessary to confirm the theory at the level of the statistical precision we have here. From the second fit we can also estimate $f_{\infty}$. We obtain

$$
f_{\infty}= \begin{cases}0.0158 \pm 0.0001 & \rho_{\min }=3.0 \\ 0.0159 \pm 0.0001 & \rho_{\min }=3.5\end{cases}
$$

in agreement with Eq. (4.41).

Finally, in order to obtain estimates of $f_{\infty}$ and $\sigma$, we have performed a fit of the form

$$
\log \left[f(\rho) \exp \left(D \rho^{\delta}\right)\right]=\log f_{\infty}+\sigma \log \rho,
$$

using the theoretical estimates for $D$ and $\delta$, for various values of $\rho \geq \rho_{\min }$ (see Table 6). These fits become rapidly unstable with increasing $\rho_{\min }$. Nonetheless, as we shall see, the final results are in reasonable agreement with the theoretical estimates even if one considers $1 \leq \rho_{\min } \leq 2$. Fitting all data with $N \geq 1000$, we obtain

$$
\begin{gathered}
\sigma= \begin{cases}0.2454 \pm 0.0003 & \rho_{\min }=1.0, \\
0.2426 \pm 0.0004 & \rho_{\min }=1.5, \\
0.2351 \pm 0.0006 & \rho_{\min }=2.0,\end{cases} \\
f_{\infty}= \begin{cases}0.01608 \pm 0.00001 & \rho_{\min }=1.0 \\
0.01612 \pm 0.00001 & \rho_{\min }=1.5 \\
0.01625 \pm 0.00002 & \rho_{\min }=2.0\end{cases}
\end{gathered}
$$

These results are not far from the theoretical estimates, although they show a trend with increasing $\rho_{\min }$ which is the opposite of what one expects theoretically: indeed the difference between the numerical and the theoretical estimates increases with 
$\rho_{\text {min }} \rightarrow \infty$. It should be noticed however that this behaviour could be a result of corrections to scaling: indeed, for $\rho_{\min }=2$, one observes that the estimates of $\sigma$ increase with $N$. If we analyze together all data with $N \geq N_{\min }$, we have $\left(\rho_{\min }=2\right)$

$$
\begin{gathered}
\sigma= \begin{cases}0.2351 \pm 0.0006 & N_{\min }=1000 \\
0.2367 \pm 0.0006 & N_{\min }=2000, \\
0.2379 \pm 0.0007 & N_{\min }=4000 \\
0.2393 \pm 0.0009 & N_{\min }=8000\end{cases} \\
f_{\infty}= \begin{cases}0.01625 \pm 0.00001 & N_{\min }=1000 \\
0.01622 \pm 0.00001 & N_{\min }=2000 \\
0.01619 \pm 0.00001 & N_{\min }=4000 \\
0.01616 \pm 0.00002 & N_{\min }=8000\end{cases}
\end{gathered}
$$

Therefore, we observe two opposite effects: $\sigma$ increases with increasing $\rho_{\text {min }}$ because of non-asymptotic corrections in $f(\rho)$, while it decreases with increasing $N_{\text {min }}$ because of corrections to scaling. Such a behaviour is not unexpected, since $f_{w}(\rho)$ was found to behave in exactly the same manner. For these reasons an accurate numerical check of the predictions for $\sigma$ and $f_{\infty}$ is difficult: it is however reassuring that all estimates are reasonably near the theoretical results.

Finally, we have studied the behaviour of $f(\rho)$ for $\rho \rightarrow 0$. In this case we have performed fits of the form

$$
\log f(\rho)=\log f_{1}+\theta \log \rho,
$$

using, in each case, only data with $\rho_{\min }<\rho<\rho_{\max }$. We have introduced here two cuts, $\rho_{\min }$ and $\rho_{\max }$. The meaning of the latter is clear: it plays the role of $\rho_{\min }$ in the analysis of the large- $\rho$ behaviour of the EEDF. The second parameter $\rho_{\text {min }}$ is introduced to eliminate spurious lattice effects. Indeed, the scaling limit is obtained taking $|\mathbf{r}|$ to infinity. In other words, small values of $r$ should be discarded. In our fits we have taken $\rho_{\min }=0.1$. Of course, this choice introduces a bias, and one should study the limit $\rho_{\min } \rightarrow 0$ to obtain the correct asymptotic behaviour. In our case, the systematic error appears to be small: indeed the estimates are stable with respect to small changes of this parameter. The results for fixed values of $N$ are reported in Table 6. The data show a small systematic variation with $N$. Using all data with $N \geq 2000$, we obtain

$$
\begin{aligned}
& \theta=\left\{\begin{array}{lr}
0.223 \pm 0.003 & \rho_{\max }=0.6 \\
0.253 \pm 0.006 & \rho_{\max }=0.4 \\
0.281 \pm 0.031 & \rho_{\max }=0.2
\end{array}\right. \\
& f_{1}= \begin{cases}0.01539 \pm 0.00004 & \rho_{\max }=0.6 \\
0.01599 \pm 0.00012 & \rho_{\max }=0.4 \\
0.01680 \pm 0.00096 & \rho_{\max }=0.2\end{cases}
\end{aligned}
$$

The estimates of $\theta$ show a systematic variation with $\rho_{\max }$, indicating the presence of strong non-asymptotic corrections. For $\rho_{\max } \lesssim 0.40, \theta$ is in reasonable agreement with the theoretical prediction. The constant $f_{1}$ shows a similar trend, approaching the theoretical value for $\rho_{\max } \lesssim 0.40$. From $f_{1}$ we can compute $f_{0}=f_{1} 2^{\theta}$. Using 
the theoretical prediction for $\theta$, we have $f_{0}=0.01853(5), 0.01925(14), 0.0202(11)$, corresponding respectively to $\rho_{\max }=0.6,0.4,0.2$. These estimates are in reasonable agreement with the theoretical results presented before that predicted $0.015 \lesssim f_{0} \lesssim$ 0.019 .

In conclusion, our Monte Carlo results confirm the theoretical results of the previous Sections. Notice that the theoretical predictions are more precise than the Monte Carlo estimates, in spite of the large statistics and of the very long walks used.

\section{Acknowledgements}

We thank Peter Grassberger for useful comments on the first draft of this work.

\section{A Definitions and properties of the basic functions}

In this Appendix we report the definitions and asymptotic expansions of the functions that appear in our two-loop computation of the end-to-end distribution function.

Function $N_{1}(x)$

We define

$$
N_{1}(x)=\int_{0}^{\infty} d t(1-x t) e^{-x t} \log t \log |1-t|
$$

The expansion of $N_{1}(x)$ for large values of $x$ is easily computed. Indeed, in this limit the relevant contribution is due to the region $t \approx 0$. It is then enough to expand $\log |1-t|$ in powers of $t$ and integrate term by term. One obtains

$$
N_{1}(x)=\sum_{n=1}^{\infty} \frac{(n-1) !}{x^{n+1}}[n \psi(n+1)+1]-\log x \sum_{n=1}^{\infty} \frac{n !}{x^{n+1}},
$$

where $\psi(x)$ is the logarithmic derivative of Euler's $\Gamma$-function. To obtain the asymptotic expansion for small values of $x$, one first notices that $N_{1}(x)$ satisfies the differential equation

$$
N_{1}^{\prime}(x)+N_{1}(x)=\frac{1}{x^{2}}\left[2-\gamma_{E}-\log x-e^{-x} \operatorname{Ei}(x)\right]
$$

where $\gamma_{E} \approx 0.5772156649$ is Euler's constant, and Ei is the exponential integral function [50]. Solving the previous equation, one obtains a different integral representation for $N_{1}(x)$ :

$$
\begin{aligned}
& N_{1}(x)=e^{-x} \int_{0}^{x} \frac{d t}{t^{2}}\left[e^{t}\left(2-\gamma_{E}-\log t\right)-\operatorname{Ei}(t)-(2+t)\left(1-\gamma_{E}-\log t\right)\right] \\
& +e^{-x}\left[\frac{2}{x}\left(\gamma_{E}+\log x\right)+\left(1-\gamma_{E}\right) \log x-\frac{1}{2} \log ^{2} x+1+\gamma_{E}-\frac{5}{12} \pi^{2}-\frac{1}{2} \gamma_{E}^{2}\right] .
\end{aligned}
$$


Using this expression it is trivial to obtain the expansion of $N_{1}(x)$ for $x \rightarrow 0$ :

$$
\begin{aligned}
N_{1}(x)= & \frac{2}{x}\left(\log x+\gamma_{E}\right)+\frac{1}{2}\left[2-2 \gamma_{E}-\gamma_{E}^{2}-\frac{5}{6} \pi^{2}-2\left(1+\gamma_{E}\right) \log x-\log ^{2} x\right] \\
& +\frac{x}{2}\left[\frac{1}{2}-\gamma_{E}+\gamma_{E}^{2}+\frac{5}{6} \pi^{2}+\left(2 \gamma_{E}-1\right) \log x+\log ^{2} x\right]+O\left(x^{2} \log x\right) .
\end{aligned}
$$

Function $N_{2}(x)$

We define

$$
N_{2}(x)=\int_{0}^{\infty} \frac{d t}{t} J_{1}(t) \int_{C} \frac{d z}{2 \pi i} \frac{z^{3 / 2} e^{x z}}{(z+1)^{2}}\left[K_{1}^{3}(\sqrt{z} t)-H_{r}(\sqrt{z} t)\right],
$$

where $C$ is a loop contour going counterclockwise around the negative real axis, $K_{1}(x)$ and $J_{1}(x)$ are Bessel functions [50, and $H_{r}(t)$ is defined by

$$
\begin{gathered}
H_{r}(t)=e^{-r t}\left[\frac{1}{t^{3}}+\frac{r}{t^{2}}+\frac{1}{2 t}\left(-\frac{3}{2}+r^{2}+3 \gamma_{E}+3 \log \frac{t}{2}\right)\right. \\
\left.-\frac{3 r}{4}+\frac{r^{3}}{6}+\frac{3 r}{2} \gamma_{E}+\frac{3 r}{2} \log \frac{t}{2}\right] .
\end{gathered}
$$

Note that $H_{r}(t)$ is such that $K_{1}^{3}(t)-H_{r}(t) \sim t \log ^{2} t$ for $|t| \rightarrow 0$.

We want now to derive the asymptotic behaviour of $N_{2}(x)$ for large and small values of $x$. Substituting $w=x z$ and $s=t / \sqrt{x}$ we can rewrite

$$
N_{2}(x)=x^{-1 / 2} \int_{0}^{\infty} \frac{d s}{s} J_{1}(s \sqrt{x}) \int_{C} \frac{d w}{2 \pi i} \frac{w^{3 / 2} e^{w}}{(w+x)^{2}}\left[K_{1}^{3}(\sqrt{w} s)-H_{r}(\sqrt{w} s)\right] .
$$

To derive the small- $x$ behaviour, we expand $J_{1}(s \sqrt{x})$ (the corresponding series converges everywhere since $J_{1}(z)$ is an entire function) obtaining

$$
N_{2}(x)=\sum_{n=0}^{\infty} \frac{(-x)^{n}}{n !(n+1) !} \int_{0}^{\infty} \frac{d s}{s}\left(\frac{s}{2}\right)^{2 n+1} \int_{C} \frac{d w}{2 \pi i} \frac{w^{3 / 2} e^{w}}{(w+x)^{2}}\left[K_{1}^{3}(\sqrt{w} s)-H_{r}(\sqrt{w} s)\right] .
$$

Now, $K_{1}(z) \sim z^{-1 / 2} e^{-z}$ for $|z| \rightarrow \infty$. Since $|\arg w|<\pi$, we can define $t=\sqrt{w} s$, and rotate the contour so that $t$ belongs to the positive real axis. We obtain

$$
N_{2}(x)=\sum_{n=0}^{\infty} \frac{(-x)^{n}}{n !(n+1) !} \int_{0}^{\infty} \frac{d t}{t}\left(\frac{t}{2}\right)^{2 n+1}\left[K_{1}^{3}(t)-H_{r}(t)\right] \int_{C} \frac{d w}{2 \pi i} \frac{w^{1-n} e^{w}}{(w+x)^{2}} .
$$

The last integral can be done exactly. We get

$$
\int_{C} \frac{d w}{2 \pi i} \frac{w^{1-n} e^{w}}{(w+x)^{2}}=(-1)^{n+1} x^{-n} e^{-x}(x+n-1)+\sum_{k=0}^{n-2} \frac{n-k-1}{k !}(-x)^{k-n},
$$


with the convention that, for $n<2$, the summation is zero. Substituting in Eq. (A.10) we obtain

$$
\begin{aligned}
N_{2}(x)= & -e^{-x} \int_{0}^{\infty} \frac{d t}{t}\left[K_{1}^{3}(t)-H_{r}(t)\right]\left[\frac{t}{2} I_{0}(t)+(x-2) I_{1}(t)\right] \\
& +\sum_{k=0}^{\infty} \frac{(-x)^{k}}{k !} \int_{0}^{\infty} \frac{d t}{t}\left[K_{1}^{3}(t)-H_{r}(t)\right] \\
& \times\left[\frac{t}{2} I_{0}(t)-(k+2) I_{1}(t)-\sum_{n=0}^{k} \frac{n-k-1}{n !(n+1) !}\left(\frac{t}{2}\right)^{2 n+1}\right] .
\end{aligned}
$$

This expression can be simplified and one obtains the final result

$$
N_{2}(x)=\sum_{k=0}^{\infty} \frac{(-x)^{k}}{k !} \sum_{n=0}^{k} \frac{k+1-n}{n !(n+1) !} \int_{0}^{\infty} \frac{d t}{t}\left[K_{1}^{3}(t)-H_{r}(t)\right]\left(\frac{t}{2}\right)^{2 n+1} .
$$

This expansion converges absolutely for all values of $x$ and allows the computation of $N_{2}(x)$ up to quite large values of $x$ with a small effort. It gives immediately the small- $x$ expansion of $N_{2}(x)$.

To compute the large- $x$ behaviour of $N_{2}(x)$, we start by introducing the Mellin transforms of $\left[K_{1}^{3}(t)-H_{r}(t)\right] / t$ and of $J_{1}(t)$. Explicitly, we define

$$
M(z) \equiv \int_{0}^{\infty} d t t^{z-2}\left[K_{1}^{3}(t)-H_{r}(t)\right]
$$

and compute

$$
\int_{0}^{\infty} d t t^{z-1} J_{1}(t)=2^{z-1} \frac{\Gamma\left(\frac{z+1}{2}\right)}{\Gamma\left(\frac{3-z}{2}\right)} .
$$

Eqs. A.14) and (A.15) are defined for $\operatorname{Re} z>0$ and $-1<\operatorname{Re} z<\frac{3}{2}$ respectively. Using the Parseval formula for Mellin transforms, we can rewrite $N_{2}(x)$ as

$$
N_{2}(x)=x^{-1 / 2} \int_{C} \frac{d w}{2 \pi i} \frac{w^{2} e^{w}}{(w+x)^{2}} \int_{\gamma-i \infty}^{\gamma+i \infty} \frac{d z}{2 \pi i} M(1-z) x^{-z / 2}(4 w)^{(z-1) / 2} \frac{\Gamma\left(\frac{z+1}{2}\right)}{\Gamma\left(\frac{3-z}{2}\right)},
$$

where $\gamma$ is any real number with $-1<\gamma<1$. Let us now show that $N_{2}(x) \rightarrow 0$ for $x \rightarrow \infty$ faster than $x^{-3+\epsilon}$, for any $\epsilon>0$. Indeed

$$
\left|N_{2}(x)\right| \leq x^{-1 / 2-\gamma / 2} 2^{\gamma-1} \int_{C} \frac{|d w|}{2 \pi} \frac{e^{\operatorname{Re} w}}{|w+x|^{2}}|w|^{(\gamma+3) / 2} f(w)
$$

where

$$
f(w) \equiv \int_{-\infty}^{\infty} \frac{d y}{2 \pi}|M(1-\gamma-i y)| e^{-y(\arg w) / 2}\left|\frac{\Gamma\left(\frac{\gamma+i y+1}{2}\right)}{\Gamma\left(\frac{3-\gamma-i y}{2}\right)}\right| .
$$

Let us first consider the integral $f(w)$. For $|y| \rightarrow \infty$, an easy computation shows that $|M(1-\gamma-i y)| \sim|y|^{p(\gamma)} e^{-\pi y / 2}$, where $p(\gamma)$ is an exponent we do not need to know explicitly. In the same limit the ratio of $\Gamma$-functions behaves as $|y|^{\gamma-1}$. Thus, the integral $f(w)$ is finite for $|\arg w|<\pi$. For $|\arg w| \rightarrow \pi$, depending on the value of $\gamma$, 
the integral may be finite or diverge as a power of $(|\arg w|-\pi)$. But, with our choice of $C,|\arg w| \rightarrow \pi$ corresponds to $\operatorname{Re} w \rightarrow-\infty$. In this limit the integrand in (A.17) decreases exponentially, therefore making the whole expression finite. Finally notice that

$$
\frac{1}{|w+x|^{2}} \leq \frac{4}{x^{2}}\left[1-\chi\left(-\frac{3 x}{2} \leq \operatorname{Re} w \leq-\frac{x}{2}\right)\right]+\frac{1}{(\operatorname{Im} w)^{2}} \chi\left(-\frac{3 x}{2} \leq \operatorname{Re} w \leq-\frac{x}{2}\right)
$$

where $\chi$ (condition) is 1 if the condition is satisfied, 0 otherwise. It follows

$$
\begin{aligned}
\left|N_{2}(x)\right| \leq & x^{-5 / 2-\gamma / 2} 2^{\gamma+1} \int_{C} \frac{|d w|}{2 \pi} e^{\operatorname{Re} w}|w|^{(\gamma+3) / 2} f(w) \\
& +x^{-1 / 2-\gamma / 2} 2^{\gamma-1} \int_{\bar{C}} \frac{|d w|}{2 \pi} \frac{e^{\operatorname{Re} w}}{(\operatorname{Im} w)^{2}}|w|^{(\gamma+3) / 2} f(w),
\end{aligned}
$$

where $\bar{C}$ is the part of $C$ with $-\frac{3 x}{2} \leq \operatorname{Re} w \leq-\frac{x}{2}$. It is possible to choose $C$ so that $|\operatorname{Im} w|$ is constant along $\bar{C}$. It is then trivial to show that the second integral in Eq. (A.20) is bounded by $x^{p} e^{-x / 2}$, where $p$ is an appropriate power. Thus $\left|N_{2}(x)\right|<$ const $x^{-5 / 2-\gamma / 2}$. Since $\gamma$ is arbitrary with $\gamma<1$, the result follows immediately. A little more work, using the technique presented for $N_{3}(x)$, allows to show that $N_{2}(x) \sim O\left(x^{-3} \log ^{2} x\right)$. It is interesting to notice that, by adding additional terms in $H_{r}(x)$, one can make $N_{2}(x)$ decrease faster: if $H_{r}(x)$ is such that $K_{1}^{3}(x)-H_{r}(x) \sim$ $O\left(x^{n} \log ^{3} x\right)$, then $N_{2}(x)$ decreases faster than $x^{-n / 2-2+\epsilon}$, for any $\epsilon>0$ (more precisely $\left.N_{2}(x) \sim O\left(x^{-n / 2-2} \log ^{3} x\right)\right)$.

Function $N_{3}(x)$

We define

$$
N_{3}(x)=\int_{0}^{\infty} \frac{d t}{t}\left[J_{1}(t)-\frac{1}{2} t+\frac{1}{16} t^{3}\right] \int_{C} \frac{d z}{2 \pi i} \frac{z^{3 / 2} e^{x z}}{(z+1)^{2}} H_{r}(\sqrt{z} t),
$$

where $C$ is a loop contour going counterclockwise around the negative real axis, $J_{1}(x)$ is a Bessel function [50] and $H_{r}(t)$ is defined in Eq. (A.7).

The small- $x$ behaviour is easily computed using the same procedure as before. We obtain

$$
N_{3}(x)=\sum_{k=2}^{\infty} \frac{(-x)^{k}}{k !} \sum_{n=2}^{k} \frac{k+1-n}{n !(n+1) !} 2^{-2 n-1} \widehat{H}_{r}(2 n+2),
$$

where $\widehat{H}_{r}(z)$ is the Mellin transform of $H_{r}(t) / t$ :

$$
\begin{aligned}
\widehat{H}_{r}(z) \equiv & \int_{0}^{\infty} d t t^{z-2} H_{r}(t) \\
= & r^{2-z}\left[r^{2} \Gamma(z-4)+r^{2} \Gamma(z-3)+\frac{1}{4} \Gamma(z-2)\left(-3+6 \gamma_{E}+2 r^{2}\right)\right. \\
& +\frac{1}{12} \Gamma(z-1)\left(-9+18 \gamma_{E}+2 r^{2}\right)+\frac{3}{2} \Gamma(z-2)(\psi(z-2)-\log 2 r) \\
& \left.\quad+\frac{3}{2} \Gamma(z-1)(\psi(z-1)-\log 2 r)\right] .
\end{aligned}
$$


This expansion converges absolutely for all values of $x$ and it gives immediately the small- $x$ expansion of $N_{3}(x)$.

Let us now compute the behaviour for large values of $x$. We rewrite $N_{3}(x)$ as

$$
N_{3}(x)=L_{1}(x)+L_{2}(x)
$$

where

$$
\begin{aligned}
L_{1}(x)= & x^{-1 / 2} \int_{0}^{\infty} \frac{d s}{s}\left[J_{1}(\sqrt{x} s)+\theta(1-\sqrt{x} s)\left(-\frac{1}{2} s x^{1 / 2}+\frac{1}{16} s^{3} x^{3 / 2}\right)\right] \\
& \times \int_{C} \frac{d w}{2 \pi i} \frac{w^{3 / 2} e^{w}}{(w+x)^{2}} H_{r}(\sqrt{w} s), \\
L_{2}(x)= & x^{-1 / 2} \int_{0}^{\infty} \frac{d s}{s} \theta(\sqrt{x} s-1)\left(-\frac{1}{2} s x^{1 / 2}+\frac{1}{16} s^{3} x^{3 / 2}\right) \\
& \times \int_{C} \frac{d w}{2 \pi i} \frac{w^{3 / 2} e^{w}}{(w+x)^{2}} H_{r}(\sqrt{w} s),
\end{aligned}
$$

and $\theta(x)$ is Heaviside's step function.

Let us first consider $L_{1}(x)$. Using the Parseval formula for Mellin transforms we obtain

$$
L_{1}(x)=x^{-1 / 2} \int_{C} \frac{d w}{2 \pi i} \frac{w^{3 / 2} e^{w}}{(w+x)^{2}} \int_{\gamma-i \infty}^{\gamma+i \infty} \frac{d z}{2 \pi i} w^{z / 2} x^{-z / 2} \widehat{H}(1-z) B(z),
$$

where

$$
B(z)=2^{z-1} \frac{\Gamma\left(\frac{z+1}{2}\right)}{\Gamma\left(\frac{3-z}{2}\right)}-\frac{1}{2} \frac{1}{z+1}+\frac{1}{16} \frac{1}{z+3},
$$

and $\gamma$ is a real number satisfying $-5<\gamma<-3$. Now, rewrite the previous expression for $L_{1}$ as

$$
\begin{aligned}
L_{1}(x)= & x^{-1 / 2} \int_{C} \frac{d w}{2 \pi i} \frac{w^{3 / 2} e^{w}}{(w+x)^{2}} \int_{\gamma-i \infty}^{\gamma+i \infty} \frac{d z}{2 \pi i} w^{z / 2} x^{-z / 2} \widehat{H}(1-z) B(z) \\
& -x^{-1 / 2} \int_{C} \frac{d w}{2 \pi i} \frac{w^{3 / 2} e^{w}}{(w+x)^{2}} \sum_{n=-3}^{0} \operatorname{Res}_{z=n}\left[w^{z / 2} x^{-z / 2} \widehat{H}(1-z) B(z)\right],
\end{aligned}
$$

where, in the first integral, $\gamma$ is real such that $0<\gamma<1$. Repeating the discussion presented for $N_{2}(x)$ one can easily show that the first integral in Eq. (A.29) behaves as $O\left(x^{-3+\epsilon}\right)$. Neglecting terms of this order, we obtain

$$
\begin{aligned}
L_{1}(x)= & \frac{x}{64}\left(-4 \log 2+4 \gamma_{E}+11\right) \int_{C} \frac{d w}{2 \pi i} \frac{e^{w}}{(w+x)^{2}} \\
& -\left(\frac{3}{64}-\frac{27}{64} \gamma_{E}+\frac{3}{8} \gamma_{E}^{2}+\frac{27}{64} \log 2+\frac{3}{8} \log ^{2} 2-\frac{3}{4} \gamma_{E} \log 2\right) \int_{C} \frac{d w}{2 \pi i} \frac{w e^{w}}{(w+x)^{2}} \\
& -\left(-\frac{27}{128}+\frac{3}{8} \gamma_{E}-\frac{3}{8} \log 2\right) \int_{C} \frac{d w}{2 \pi i} \frac{w e^{w}}{(w+x)^{2}} \log (w / x) .
\end{aligned}
$$


Using Eq. (A.11) and $(n \geq 0)$

$$
\int_{C} \frac{d w}{2 \pi i} \frac{w^{n} e^{w}}{(w+x)^{2}} \log w=\frac{(-1)^{n+1} n !}{x^{2}}\left(1+\frac{2(n+1)}{x}\right)+O\left(x^{-4}\right),
$$

we obtain

$$
L_{1}(x)=\left(\frac{27}{128}-\frac{3}{8} \gamma_{E}+\frac{3}{8} \log 2\right) \frac{1}{x^{2}}+O\left(x^{-3} \log x\right) .
$$

The computation of the asymptotic behaviour of $L_{2}(x)$ is completely analogous. We have, discarding terms of order $x^{-3} \log ^{2} x$,

$$
L_{2}(x)=-x^{-1 / 2} \int_{C} \frac{d w}{2 \pi i} \frac{w^{3 / 2} e^{w}}{(w+x)^{2}} \sum_{n=-3}^{0} \operatorname{Res}_{z=n}\left[w^{z / 2} x^{-z / 2} \widehat{H}(1-z)\left(\frac{1}{2} \frac{1}{z+1}-\frac{1}{16} \frac{1}{z+3}\right)\right] .
$$

Using the previous results and $(n \geq 0)$

$$
\int_{C} \frac{d w}{2 \pi i} \frac{w^{n} e^{w}}{(w+x)^{2}} \log ^{2} w=\frac{2(-1)^{n+1} \psi(n+1) n !}{x^{2}}+O\left(x^{-3}\right)
$$

we obtain

$$
L_{2}(x)=\frac{1}{32 x^{2}}\left[x+\frac{5}{4}+6 \gamma_{E}-12 \log 2-6 \log x\right]+O\left(x^{-3} \log ^{2} x\right) .
$$

Summing up, we have

$$
N_{3}(x)=\frac{1}{32 x^{2}}\left[x+8-6 \gamma_{E}-6 \log x\right]+O\left(x^{-3} \log ^{2} x\right) .
$$

Fourier transform of $\widehat{N}_{2}\left(Q^{2}\right)+N_{3}\left(Q^{2}\right)$

We want now to report the small- $\rho$ behaviour of the Fourier transform of $\widehat{N}_{2}\left(Q^{2}\right)+$ $N_{3}\left(Q^{2}\right)$ that we use in Sec. 3. We define

$$
N(\rho) \equiv \int \frac{d^{4} Q}{(2 \pi)^{4}} e^{i \mathbf{Q} \cdot \rho}\left(\widehat{N}_{2}\left(Q^{2}\right)+N_{3}\left(Q^{2}\right)\right)
$$

where, cf. Eq. (3.15),

$$
\widehat{N}_{2}(x)=N_{2}(x)-e^{-x}(1+x) N_{2}(0)-e^{-x} N_{2}^{\prime}(0) .
$$

Using the definitions of $N_{2}(x)$ and $N_{3}(x)$ we can rewrite

$$
\widehat{N}_{2}(x)+N_{3}(x)=x^{-1 / 2} \int_{C} \frac{d w}{2 \pi i} \frac{w^{3 / 2} e^{w}}{(w+x)^{2}} \int_{0}^{\infty} \frac{d s}{s} K_{1}^{3}(s \sqrt{w})\left[J_{1}(\sqrt{x} s)-\frac{1}{2} \sqrt{x} s+\frac{1}{16} x^{3 / 2} s^{3}\right] .
$$

Using the fact that, for any function $h\left(Q^{2}\right)$, we have

$$
\int \frac{d^{4} Q}{(2 \pi)^{4}} e^{i \mathbf{Q} \cdot \rho} h\left(Q^{2}\right)=\frac{1}{4 \pi^{2} \rho} \int_{0}^{\infty} d Q Q^{2} J_{1}(Q \rho) h\left(Q^{2}\right)
$$


we can perform the integral over $Q$, cf. formula 6.541 of Ref. [50], obtaining

$$
\begin{aligned}
N(\rho)= & \frac{1}{16 \pi^{2} \rho} \int_{C} \frac{d w}{2 \pi i} w e^{w}\left\{\int _ { 0 } ^ { \infty } \frac { d s } { s } K _ { 1 } ^ { 3 } ( s ) \left[-\frac{s}{\sqrt{w}} K_{1}(\sqrt{w} \rho)\left(I_{2}(s)+I_{0}(s)-1-\frac{3}{8} s^{2}\right)\right.\right. \\
& \left.\left.+\left(K_{2}(\sqrt{w} \rho)+K_{0}(\sqrt{w} \rho)\right)\left(I_{1}(s)-\frac{1}{2} s-\frac{1}{16} s^{3}\right)\right]\right\} \\
& +\frac{1}{16 \pi^{2} \rho} \int_{C} \frac{d w}{2 \pi i} w e^{w}\left\{-\rho\left[I_{0}(\rho \sqrt{w})+I_{2}(\rho \sqrt{w})\right] \int_{\rho}^{\infty} \frac{d s}{s} K_{1}^{4}(s \sqrt{w})\right. \\
& -\rho\left[K_{0}(\rho \sqrt{w})+K_{2}(\rho \sqrt{w})\right] \int_{\rho}^{\infty} \frac{d s}{s} K_{1}^{3}(s \sqrt{w}) I_{1}(s \sqrt{w}) \\
& +K_{1}(\rho \sqrt{w}) \int_{\rho}^{\infty} d s K_{1}^{3}(s \sqrt{w})\left[I_{2}(s \sqrt{w})+I_{0}(s \sqrt{w})\right] \\
& \left.+I_{1}(\rho \sqrt{w}) \int_{\rho}^{\infty} d s K_{1}^{3}(s \sqrt{w})\left[K_{2}(s \sqrt{w})+K_{0}(s \sqrt{w})\right]\right\} .
\end{aligned}
$$

Expanding for $\rho \rightarrow 0$, after a lengthy calculation, we obtain

$$
\begin{aligned}
N(\rho) \approx & \frac{1}{128 \pi^{2} \rho^{2}}-\frac{3}{128 \pi^{2}} \log ^{2} \frac{\rho}{2}-\frac{1}{128 \pi^{2}}\left(1+3 \gamma_{E}\right) \log \frac{\rho}{2} \\
& +\frac{5}{1024}-\frac{3}{256 \pi^{2}}-\frac{\gamma_{E}}{256 \pi^{2}}-\frac{3}{512 \pi^{2}} \gamma_{E}^{2}+\frac{A}{32 \pi^{2}}+O\left(\rho^{2} \log ^{2} \rho\right)
\end{aligned}
$$

where

$$
A=\int_{0}^{\infty} d s\left[K_{1}^{3}(s)\left(1+\frac{1}{4} s^{2}\right)-e^{-s / 2}\left(\frac{1}{s^{3}}+\frac{1}{2 s^{2}}+\frac{3}{2 s} \gamma_{E}-\frac{3}{8 s}+\frac{3}{2 s} \log \frac{s}{2}\right)\right] .
$$

Numerically $A \approx-1.30204$.

Function $g_{1}(x)$

We define

$$
g_{1}(x)=\frac{1}{2}\left(1-e^{-x}\right)+\frac{1}{2}(1-x) e^{-x}(\operatorname{Ei}(x)-\log x),
$$

where $\operatorname{Ei}(x)$ is the exponential integral function [50]. We are interested in the behaviour of $g_{1}(x)$ for large and small values of $x$. These expansions are easily obtained using the corresponding results for $\operatorname{Ei}(x)$. For $x \ll 1$ we obtain

$$
\begin{aligned}
g_{1}(x) & =-\frac{1}{2} \sum_{n=0}^{\infty} \frac{(n+1) \psi(n+1)}{n !}(-x)^{n} \\
& =\frac{1}{2} \gamma_{E}+\left(1-\gamma_{E}\right) x+\frac{3}{8}\left(2 \gamma_{E}-3\right) x^{2}+O\left(x^{3}\right)
\end{aligned}
$$

For $x \gg 1$ we have the asymptotic expansion

$$
g_{1}(x)=-\frac{1}{2} \sum_{n=0}^{\infty} \frac{(n+1)(n+1) !}{x^{2+n}} .
$$


Function $g_{2}(x)$

We define

$$
\begin{aligned}
g_{2}(x)= & \frac{1}{4 x}\left[e^{-x} \operatorname{Ei}(x)+\gamma_{E}+\log x\right]-\frac{1}{4} \log x \\
& +\frac{1}{8} e^{-x}(1-x) \log x[\log x-2 \operatorname{Ei}(x)] \\
& +\frac{1}{8} e^{-x}\left[2 \log x-4 \operatorname{Ei}(x)-\pi^{2}(1-x)\right]-\frac{1}{4}(1-x) N_{1}(x) .
\end{aligned}
$$

For $x \ll 1$ we have

$$
\begin{aligned}
g_{2}(x) & =\frac{1}{8} \sum_{n=0}^{\infty}(n+1) \frac{\psi^{2}(n+1)-\psi^{\prime}(n+1)}{n !}(-x)^{n} \\
& =\frac{1}{8}\left(\gamma_{E}^{2}-\frac{1}{6} \pi^{2}\right)+\frac{x}{4}\left(-2+2 \gamma_{E}-\gamma_{E}^{2}+\frac{1}{6} \pi^{2}\right)+O\left(x^{2}\right) .
\end{aligned}
$$

For $x \gg 1$ we have

$$
g_{2}(x)=\frac{1}{4} \sum_{k=1}^{\infty} \frac{k ! k \psi(k+1)}{x^{k+1}} .
$$

Function $g_{3}(x)$

We define

$$
g_{3}(x)=-1+x e^{-x} \operatorname{Ei}(x)+e^{-x}(1-x \log x) .
$$

For $x \ll 1$ we have

$$
\begin{aligned}
g_{3}(x) & =\sum_{n=1}^{\infty} \frac{\psi(n+1)}{(n-1) !}(-x)^{n} \\
& =x\left(\gamma_{E}-1\right)+\frac{x^{2}}{2}\left(3-2 \gamma_{E}\right)++O\left(x^{3}\right) .
\end{aligned}
$$

For $x \gg 1$ we obtain

$$
g_{3}(x)=\sum_{k=1}^{\infty} \frac{k !}{x^{k}} .
$$

\section{References}

[1] P. J. Flory, Principles of Polymer Chemistry (Cornell University Press, Ithaca, NY, 1953).

[2] P. G. de Gennes, Scaling Concepts in Polymer Physics (Cornell University Press, Ithaca, NY, 1979).

[3] J. des Cloizeaux and G. Jannink, Les Polymères en Solution (Les Editions de Physique, Les Ulis, 1987); English translation: Polymers in Solution: Their Modeling and Structure (Oxford University Press, Oxford-New York, 1990). 
[4] K. F. Freed, Renormalization-Group Theory of Macromolecules (John Wiley, New York, 1987).

[5] P. G. de Gennes, Phys. Lett. A38, 339 (1972).

[6] M. E. Fisher and B. J. Hiley, J. Chem. Phys. 34, 1253 (1961).

[7] M. E. Fisher, J. Chem. Phys. 44, 616 (1966).

[8] J. Mazur, J. Res. Natl. Bur. Stand. A69, 355 (1965); J. Chem. Phys. 43, 4354 (1965).

[9] D. S. Mc Kenzie and M. A. Moore, J. Phys. A4, L82 (1971).

[10] D. S. Mc Kenzie, Phys. Rep. 27C, 35 (1976).

[11] J. des Cloizeaux, Phys. Rev. A10, 1665 (1974); J. Physique 41, 223 (1980).

[12] P. H. Verdier and W. H. Stockmayer, J. Chem. Phys. 36, 227 (1962).

[13] F. T. Wall, S. Windwer, and R. J. Gans, J. Chem. Phys. 38, 2220 (1963).

[14] A. Baumgärtner, Z. Phys. 42, 265 (1981).

[15] M. Bishop and J. H. R. Clarke, J. Chem. Phys. 94, 3936 (1991).

[16] M. Bishop, J. H. R. Clarke, A. Rey, and J. J. Freire, J. Chem. Phys. 95, 4589 (1991).

[17] J. Dayantis and J.-F. Palierne, J. Chem. Phys. 95, 6088 (1991).

[18] N. Eizenberg and J. Klafter, J. Chem. Phys. 99, 3976 (1993).

[19] J. S. Pedersen, M. Laso, and P. Schurtenberger, Phys. Rev. E54, R5917 (1996).

[20] J. P. Valleau, J. Chem. Phys. 104, 3071 (1996).

[21] The different proposals appearing in the literature and many old numerical results have been reviewed by Bishop and Clarke [15].

[22] Y. Oono, T. Ohta, and K. F. Freed, Macromolecules 14, 880 (1981); J. Chem. Phys. 74, 6458 (1981).

[23] In continuum models, the function $c_{N}(\mathbf{r})$ is not well defined. For models regularized on a lattice, $c_{N}(\mathbf{r})$ can be identified with the number of walks starting at the origin and ending in $\mathbf{r}$.

[24] P. Belohorec and B. G. Nickel, Accurate universal and two-parameter model results from a Monte-Carlo renormalization group study, University of Guelph report (September 1997), unpublished. 
[25] S. Caracciolo, M. S. Causo and A. Pelissetto, Phys. Rev. E57, R1215 (1998), cond-mat/9703250.

[26] S. F. Edwards, Proc. Phys. Soc. 85, 613 (1965).

[27] Y. Oono, in Advances in Chemical Physics, Vol. LXI, I. Prigogine and S. A. Rice eds., pag. 301, 1985.

[28] The reader should notice that we are not obtaining a three-loop determination of $\widetilde{f}_{\infty}$ by means of a two-loop computation. The order $\epsilon^{3}$ contribution is obtained from the two-loop expression of $f_{0}$ and the three-loop results for the critical exponents 29.

[29] E. Brézin, J. C. Le Guillou, J. Zinn-Justin, and B. G. Nickel, Phys. Lett. 44A, 227 (1973).

[30] J. C. Le Guillou and J. Zinn-Justin, Phys. Rev. Lett. 39, 95 (1977); Phys. Rev. B21, 3976 (1980).

[31] The resummation procedure depends on two free parameters, $\alpha$ and $b$ in the notation of Ref. [30]. The reported errors are related to the variation of the estimates with changes of $\alpha$ and $b$.

[32] M. E. Fisher and R. J. Burford, Phys. Rev. 156, 583 (1967).

[33] M. E. Fisher and A. Aharony, Phys. Rev. Lett. 31, 1238 (1973); Phys. Rev. B7, 2818 (1974).

[34] A. J. Bray, Phys. Rev. B 14, 1248 (1976).

[35] M. Campostrini, A. Pelissetto, P. Rossi, and E. Vicari, Europhys. Lett. 38, 577 (1997); Phys. Rev. E57, 184 (1998).

[36] The coupling constant $g$ can be expressed in terms of the second virial coefficient for a polydisperse ensemble of polymers, see [37.

[37] M. Muthukumar and B. G. Nickel, J. Chem. Phys. 80, 5839 (1984); 86, 460 (1987).

[38] G. A. Baker, Jr., B. G. Nickel and D. I. Meiron, Phys. Rev. Lett. 36, 1351 (1977); Phys. Rev. B17, 1365 (1977).

[39] D. B. Murray and B. G. Nickel, Revised estimates for critical exponents for the continuum n-vector model in 3 dimensions, University of Guelph preprint (1991), unpublished.

[40] A. Pelissetto and E. Vicari, Nucl. Phys. B519 [FS], 626 (1998); the estimates appearing in this work are affected by a small numerical error. Revised estimates appear in: The effective potential of $N$-vector models: a field-theoretic study to $O\left(\epsilon^{3}\right)$, cond-mat/9911452. 
[41] M. Campostrini, A. Pelissetto, P. Rossi, and E. Vicari, Phys. Rev. B54, 7301 (1996).

[42] M. E. Fisher and J. S. Langer, Phys. Rev. Lett. 20, 665 (1968).

[43] E. Brézin, D. Amit, and J. Zinn-Justin, Phys. Rev. Lett. 32, 151 (1974).

[44] M. Lal, Molec. Phys. 17, 57 (1969).

[45] B. MacDonald, N. Jan, D. L. Hunter, and M. O. Steinitz, J. Phys. A18, 2627 (1985).

[46] N. Madras and A. D. Sokal, J. Stat. Phys. 50, 109 (1988).

[47] B. Li, N. Madras, and A. D. Sokal, J. Stat. Phys. 80, 661 (1995).

[48] The most precise Monte Carlo estimate of $\Delta$ is $\Delta=0.517 \pm 0.007+0.010$ [24]. Perturbative field theory gives a lower estimate [49]: $\Delta=0.478 \pm 0.010(d=3$ expansion) and $\Delta=0.486 \pm 0.016$ ( $\epsilon$-expansion).

[49] R. Guida and J. Zinn-Justin, J. Phys. A31, 8103 (1998).

[50] I. S. Gradshteyn and I. M. Ryzhik, Table of Integrals, Series, and Products, fourth edition (Academic Press, San Diego, 1980). 


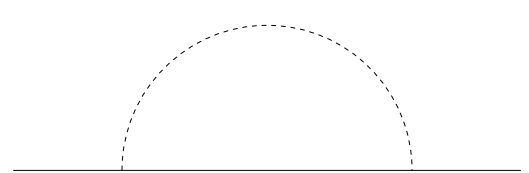

(a)

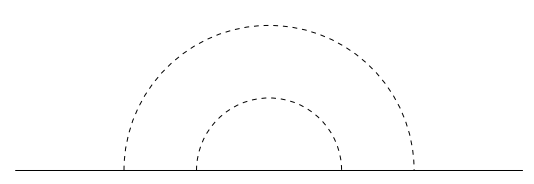

(b)

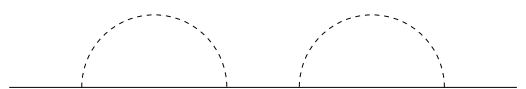

(c)

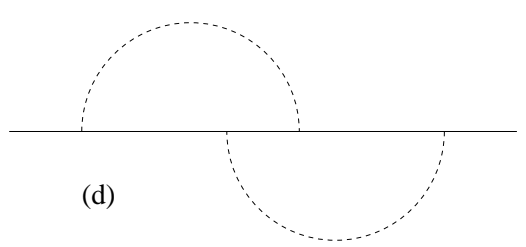

Figure 1: Graphs contributing to the EEDF to order $\epsilon^{2}$.

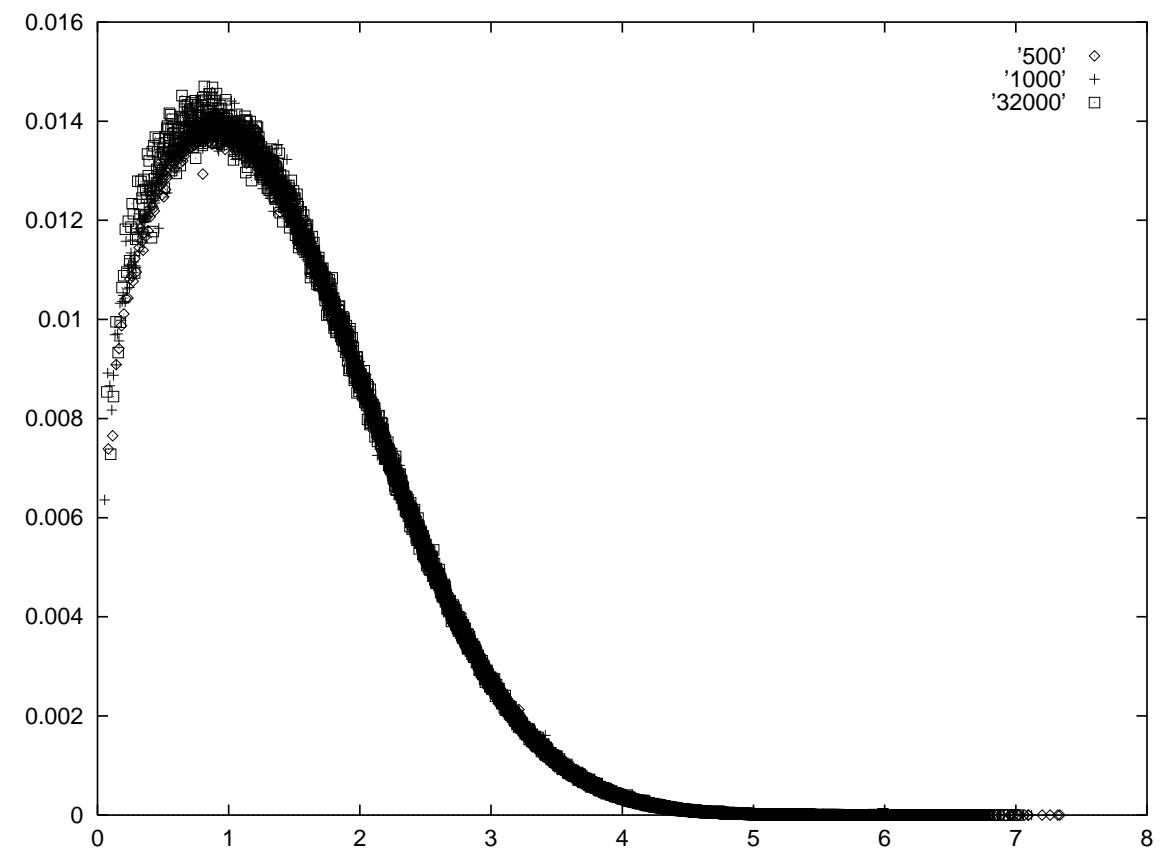

Figure 2: Plot of $f(\rho)$ for different values of $N$. 


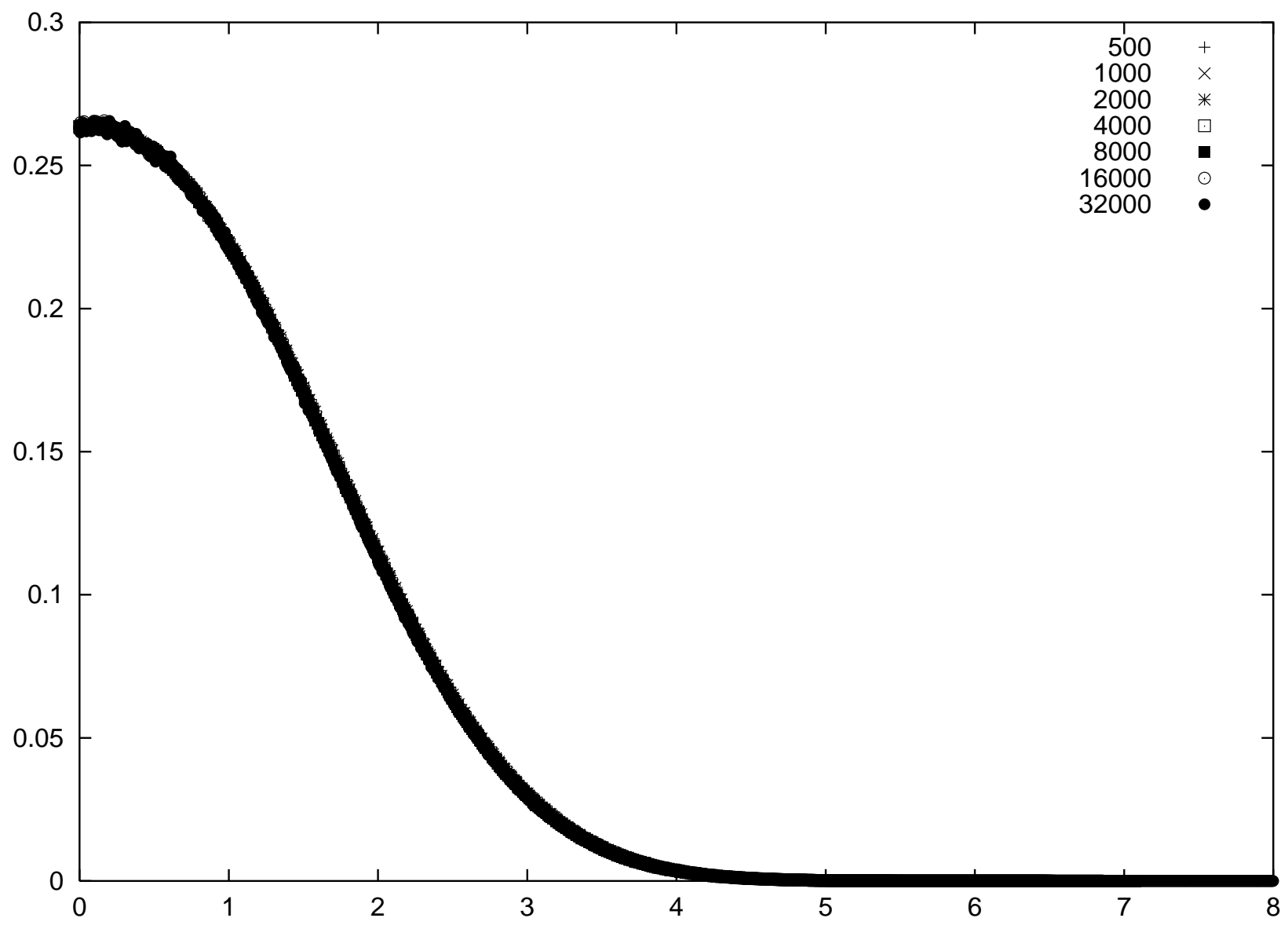




\begin{tabular}{|c|c|c|c|c|}
\hline$N$ & $M_{4}$ & $M_{6}$ & $M_{8}$ & $M_{10}$ \\
\hline 500 & $1.49998 \pm 0.00042$ & $2.9413 \pm 0.0024$ & $7.044 \pm 0.011$ & $19.785 \pm 0.057$ \\
1000 & $1.50457 \pm 0.00035$ & $2.9666 \pm 0.0020$ & $7.1600 \pm 0.0098$ & $20.306 \pm 0.050$ \\
2000 & $1.50755 \pm 0.00034$ & $2.9833 \pm 0.0019$ & $7.2360 \pm 0.0096$ & $20.647 \pm 0.050$ \\
4000 & $1.50939 \pm 0.00035$ & $2.9933 \pm 0.0020$ & $7.2811 \pm 0.0095$ & $20.849 \pm 0.050$ \\
8000 & $1.51084 \pm 0.00026$ & $3.0011 \pm 0.0015$ & $7.3153 \pm 0.0075$ & $20.996 \pm 0.039$ \\
16000 & $1.51169 \pm 0.00043$ & $3.0053 \pm 0.0026$ & $7.334 \pm 0.012$ & $21.091 \pm 0.066$ \\
32000 & $1.51340 \pm 0.00068$ & $3.0158 \pm 0.0042$ & $7.386 \pm 0.023$ & $21.34 \pm 0.11$ \\
\hline$M^{*}$ & $1.51406 \pm 0.00089$ & $3.018 \pm 0.005$ & $7.387 \pm 0.023$ & $21.34 \pm 0.13$ \\
$\mathrm{~B}$ & $-(0.00314 \pm 0.00084)$ & $-(0.016 \pm 0.0046)$ & $-(0.070 \pm 0.021)$ & $-(0.32 \pm 0.11)$ \\
$\Delta$ & $0.538 \pm 0.079$ & $0.555 \pm 0.085$ & $0.572 \pm 0.092$ & $0.56 \pm 0.11$ \\
$\mathrm{CL}$ & $70 \%$ & $66 \%$ & $67 \%$ & $66 \%$ \\
\hline th & $1.51397 \pm 0.00079$ & $3.018 \pm 0.004$ & $7.392 \pm 0.015$ & $21.35 \pm 0.06$ \\
\hline ph & $1.50876 \pm 0.00023$ & $2.993 \pm 0.001$ & $7.292 \pm 0.005$ & $20.94 \pm 0.02$ \\
\hline
\end{tabular}

Table 1: Monte Carlo results for the invariant ratios $M_{2 k}$. "CL" is the confidence level of the fit $M_{2 k}=M_{2 k}^{*}+B_{2 k}(N / 8000)^{-\Delta}$. "th" is the theoretical prediction (4.51) and "ph" the phenomenological prediction (4.52).

\begin{tabular}{|c|c|c|c|c|}
\hline & $R_{4}$ & $R_{6}$ & $R_{8}$ & $R_{10}$ \\
\hline 500 & $0.60052 \pm 0.00038$ & $0.42958 \pm 0.00080$ & $0.3348 \pm 0.0013$ & $0.2748 \pm 0.0019$ \\
1000 & $0.60023 \pm 0.00032$ & $0.42896 \pm 0.00068$ & $0.3338 \pm 0.0011$ & $0.2731 \pm 0.0016$ \\
2000 & $0.60008 \pm 0.00030$ & $0.42872 \pm 0.00065$ & $0.3336 \pm 0.0010$ & $0.2732 \pm 0.0015$ \\
4000 & $0.59983 \pm 0.00031$ & $0.42811 \pm 0.00064$ & $0.3325 \pm 0.0010$ & $0.2713 \pm 0.0015$ \\
8000 & $0.60005 \pm 0.00024$ & $0.42866 \pm 0.00050$ & $0.33339 \pm 0.00080$ & $0.2727 \pm 0.0012$ \\
16000 & $0.59994 \pm 0.00038$ & $0.42833 \pm 0.00079$ & $0.3328 \pm 0.0013$ & $0.2720 \pm 0.0019$ \\
32000 & $0.60028 \pm 0.00065$ & $0.4295 \pm 0.0012$ & $0.3351 \pm 0.0024$ & $0.2756 \pm 0.0035$ \\
\hline th & $\frac{3}{5}=0.6$ & $\frac{3}{7}=0.42857142$ & $\frac{1}{3}=0.33333333$ & $\frac{3}{11}=0.27272727$ \\
\hline
\end{tabular}

Table 2: Monte Carlo results for the invariant ratio $R_{2 k}$. "th" is the theoretical prediction. 


\begin{tabular}{|c|c||c|c|c||c|c|c|}
\hline$N$ & \multicolumn{1}{|c||}{$f_{w}(\rho)$} & \multicolumn{3}{|c|}{$f_{w}(\rho) \cdot \rho^{0.169}$} \\
\hline & & $\rho_{\min }=3$ & $\rho_{\min }=3.5$ & $\rho_{\min }=4$ & $\rho_{\min }=3$ & $\rho_{\min }=3.5$ & $\rho_{\min }=4$ \\
\hline 500 & $\delta$ & $2.489(16)$ & $2.480(38)$ & $2.54(10)$ & $2.535(17)$ & $2.515(38)$ & $2.57(10)$ \\
& $\mathrm{D}$ & $0.1328(38)$ & $0.1350(94)$ & $0.121(24)$ & $0.1203(35)$ & $0.1246(87)$ & $0.113(22)$ \\
& $f_{w, \infty}$ & $0.2395(55)$ & $0.244(17)$ & $0.216(49)$ & $0.2618(58)$ & $0.271(18)$ & $0.245(54)$ \\
\hline 1000 & $\delta$ & $2.434(13)$ & $2.388(30)$ & $2.415(73)$ & $2.479(13)$ & $2.423(30)$ & $2.444(74)$ \\
& $\mathrm{D}$ & $0.1453(33)$ & $0.1583(87)$ & $0.150(21)$ & $0.1318(30)$ & $0.1462(81)$ & $0.141(20)$ \\
& $f_{w, \infty}$ & $0.2520(47)$ & $0.276(16)$ & $0.260(45)$ & $0.2747(50)$ & $0.305(17)$ & $0.293(50)$ \\
\hline 2000 & $\delta$ & $2.420(13)$ & $2.434(29)$ & $2.476(84)$ & $2.463(13)$ & $2.468(29)$ & $2.494(83)$ \\
& $\mathrm{D}$ & $0.1483(34)$ & $0.1442(78)$ & $0.133(21)$ & $0.1346(31)$ & $0.1336(72)$ & $0.127(20)$ \\
& $f_{w, \infty}$ & $0.2506(48)$ & $0.244(13)$ & $0.224(43)$ & $0.2735(50)$ & $0.272(14)$ & $0.259(48)$ \\
\hline 4000 & $\delta$ & $2.407(13)$ & $2.447(29)$ & $2.456(74)$ & $2.452(13)$ & $2.483(28)$ & $2.486(75)$ \\
& $\mathrm{D}$ & $0.1517(33)$ & $0.1408(75)$ & $0.138(20)$ & $0.1375(31)$ & $0.1297(68)$ & $0.129(19)$ \\
& $f_{w, \infty}$ & $0.2541(47)$ & $0.235(12)$ & $0.232(40)$ & $0.2767(50)$ & $0.261(13)$ & $0.262(44)$ \\
\hline 8000 & $\delta$ & $2.4139(98)$ & $2.419(23)$ & $2.450(58)$ & $2.4590(99)$ & $2.454(23)$ & $2.480(59)$ \\
& $\mathrm{D}$ & $0.1490(25)$ & $0.1477(61)$ & $0.139(15)$ & $0.1350(23)$ & $0.1362(57)$ & $0.129(15)$ \\
& $f_{w, \infty}$ & $0.2464(35)$ & $0.244(10)$ & $0.224(30)$ & $0.2684(37)$ & $0.271(11)$ & $0.253(33)$ \\
\hline 16000 & $\delta$ & $2.370(21)$ & $2.423(51)$ & $2.36(15)$ & $2.414(21)$ & $2.458(51)$ & $2.37(15)$ \\
& $\mathrm{D}$ & $0.1617(61)$ & $0.147(14)$ & $0.167(48)$ & $0.1465(55)$ & $0.136(13)$ & $0.160(45)$ \\
& $f_{w, \infty}$ & $0.2658(86)$ & $0.241(22)$ & $0.29(10)$ & $0.2883(88)$ & $0.268(24)$ & $0.33(11)$ \\
\hline 32000 & $\delta$ & $2.433(32)$ & $2.382(83)$ & $1.69(22)$ & $2.482(33)$ & $2.422(83)$ & $1.74(22)$ \\
& $\mathrm{D}$ & $0.1435(79)$ & $0.157(24)$ & $0.62(28)$ & $0.1292(73)$ & $0.144(22)$ & $0.56(25)$ \\
& $f_{w, \infty}$ & $0.236(10)$ & $0.257(39)$ & $2.4(2.3)$ & $0.257(11)$ & $0.282(41)$ & $2.3(2.1)$ \\
\hline
\end{tabular}

Table 3: Results for the fit $g(\rho)=f_{w, \infty} \exp \left(-D \rho^{\delta}\right)$ for $\rho>\rho_{\min }$. The first three columns refer to $g(\rho)=f_{w, M C}(\rho)$, the last three columns to $g(\rho)=f_{w, M C}(\rho) \rho^{0.169}$. Here $f_{w, M C}(\rho)$ is the Monte Carlo "wall-to-wall" EEDF.

\begin{tabular}{|c|c||c|c|c|}
\hline$N$ & & $\rho_{\min }=2$. & $\rho_{\min }=2.5$ & $\rho_{\min }=3$. \\
\hline 500 & $\sigma_{w}$ & $-0.1153(31)$ & $-0.1752(63)$ & $-0.252(12)$ \\
& $f_{w, \infty}$ & $0.27406(81)$ & $0.2934(20)$ & $0.3232(50)$ \\
\hline 1000 & $\sigma_{w}$ & $-0.12149(24)$ & $-0.1641(47)$ & $-0.234(10)$ \\
& $f_{w, \infty}$ & $0.27204(62)$ & $0.2855(15)$ & $0.3117(38)$ \\
\hline 2000 & $\sigma_{w}$ & $-0.1254(24)$ & $-0.1527(45)$ & $-0.1902(97)$ \\
& $f_{w, \infty}$ & $0.27076(60)$ & $0.2792(14)$ & $0.2926(35)$ \\
\hline 4000 & $\sigma_{w}$ & $-0.1268(22)$ & $-0.1513(44)$ & $-0.1954(94)$ \\
& $f_{w, \infty}$ & $0.26937(56)$ & $0.2770(13)$ & $0.2927(34)$ \\
\hline 8000 & $\sigma_{w}$ & $-0.1280(17)$ & $-0.1417(34)$ & $-0.1537(72)$ \\
& $f_{w, \infty}$ & $0.26850(44)$ & $0.2727(10)$ & $0.2768(24)$ \\
\hline 16000 & $\sigma_{w}$ & $-0.1314(34)$ & $-0.1473(70)$ & $-0.2001(17)$ \\
& $f_{w, \infty}$ & $0.26874(87)$ & $0.2735(21)$ & $0.2919(60)$ \\
\hline 32000 & $\sigma_{w}$ & $-0.1087(51)$ & $-0.125(11)$ & $-0.090(33)$ \\
& $f_{w, \infty}$ & $0.2624(12)$ & $0.2670(33)$ & $0.256(10)$ \\
\hline
\end{tabular}

Table 4: Results for the fit $f_{w, M C}(\rho) \exp \left(D \rho^{\delta}\right)=f_{w, \infty} \rho^{\sigma_{w}}$ for $\rho>\rho_{\min } \cdot f_{w, M C}(\rho)$ is the Monte Carlo "wall-to-wall" EEDF. $D$ and $\delta$ have been set equal to the theoretical predictions, Eqs. (2.9) and (4.32). 


\begin{tabular}{|c|c|c|c|c|c|c|c|c|}
\hline \multirow[t]{2}{*}{$N$} & \multirow[t]{2}{*}{$N_{s h}$} & & \multicolumn{3}{|c|}{$f_{M C}(\rho)$} & \multicolumn{3}{|c|}{$f_{M C}(\rho) \cdot \rho^{-0.255}$} \\
\hline & & & $\rho_{\min }=3$ & $\rho_{\min }=3.5$ & $\rho_{\min }=4$ & $\rho_{\min }=3$ & $\rho_{\min }=3.5$ & $\rho_{\min }=4$ \\
\hline \multirow[t]{6}{*}{500} & \multirow[t]{3}{*}{1} & $\delta$ & $2.5368(59)$ & $2.479(11)$ & $2.296(23)$ & $2.4733(58)$ & $2.430(11)$ & $2.260(23)$ \\
\hline & & $\mathrm{D}$ & $0.1170(12)$ & $0.1305(27)$ & $0.1877(86)$ & $0.1346(14)$ & $0.1461(30)$ & $0.2050(93)$ \\
\hline & & $f_{\infty}$ & $0.01827(15)$ & $0.02045(43)$ & $0.0333(21)$ & $0.01583(14)$ & $0.01732(38)$ & $0.0279(18)$ \\
\hline & \multirow[t]{3}{*}{10} & $\delta$ & $2.5662(59)$ & $2.545(11)$ & $2.464(23)$ & $2.5019(58)$ & $2.495(11)$ & $2.425(23)$ \\
\hline & & $\mathrm{D}$ & $0.1113(12)$ & $0.1158(24)$ & $0.1359(61)$ & $0.1282(13)$ & $0.1299(26)$ & $0.1490(66)$ \\
\hline & & $f_{\infty}$ & $0.01762(14)$ & $0.01833(36)$ & $0.0224(12)$ & $0.01523(13)$ & $0.01545(32)$ & $0.0185(11)$ \\
\hline \multirow[t]{6}{*}{1000} & \multirow[t]{3}{*}{1} & $\delta$ & $2.4892(47)$ & $2.4078(88)$ & $2.202(18)$ & $2.4268(46)$ & $2.3601(89)$ & $2.167(18)$ \\
\hline & & $\mathrm{D}$ & $0.1262(10)$ & $0.1473(24)$ & $0.2216(81)$ & $0.1450(12)$ & $0.1646(27)$ & $0.2419(88)$ \\
\hline & & $f_{\infty}$ & $0.01905(13)$ & $0.02244(39)$ & $0.0395(21)$ & $0.01655(12)$ & $0.01911(35)$ & $0.0335(19)$ \\
\hline & \multirow[t]{3}{*}{20} & $\delta$ & $2.5336(47)$ & $2.5094(88)$ & $2.461(19)$ & $2.4698(46)$ & $2.4596(87)$ & $2.422(18)$ \\
\hline & & $\mathrm{D}$ & $0.11696(96)$ & $0.1224(20)$ & $0.1345(51)$ & $0.1346(11)$ & $0.1373(21)$ & $0.1477(52)$ \\
\hline & & $f_{\infty}$ & $0.01800(12)$ & $0.01885(30)$ & $0.02107(97)$ & $0.01560(11)$ & $0.01593(27)$ & $0.01744(79)$ \\
\hline \multirow{6}{*}{2000} & \multirow[t]{3}{*}{40} & $\delta$ & $2.5123(44)$ & $2.4940(83)$ & $2.421(17)$ & $2.4489(43)$ & $2.4442(81)$ & $\begin{array}{l}2.382(17) \\
\end{array}$ \\
\hline & & $\mathrm{D}$ & $0.12072(94)$ & $0.1249(19)$ & $0.1443(48)$ & $0.1389(11)$ & $0.1401(21)$ & $0.1585(53)$ \\
\hline & & $f_{\infty}$ & $0.01822(12)$ & $0.01885(29)$ & $0.02257(95)$ & $0.01580(10)$ & $0.01595(25)$ & $0.01875(82)$ \\
\hline & \multirow[t]{3}{*}{80} & $\delta$ & $2.5161(44)$ & $2.5035(83)$ & $2.445(17)$ & $2.4526(43)$ & $2.4534(83)$ & $2.406(17)$ \\
\hline & & $\mathrm{D}$ & $0.11993(94)$ & $0.1228(19)$ & $0.1376(46)$ & $0.1380(11)$ & $0.1378(21)$ & $0.1513(50)$ \\
\hline & & $f_{\infty}$ & $0.01813(11)$ & $0.01856(28)$ & $0.02135(89)$ & $0.01573(10)$ & $0.01569(25)$ & $0.01771(76)$ \\
\hline \multirow[t]{6}{*}{4000} & \multirow[t]{3}{*}{100} & $\delta$ & $2.5031(42)$ & $2.4716(79)$ & $2.4289(16)$ & $2.4398(41)$ & $2.4221(79)$ & $2.390(17)$ \\
\hline & & $\mathrm{D}$ & $0.12229(91)$ & $0.1297(19)$ & $0.1413(45)$ & $0.1407(10)$ & $0.1455(22)$ & $0.1552(52)$ \\
\hline & & $f_{\infty}$ & $0.01828(11)$ & $0.01940(29)$ & $0.02168(85)$ & $0.01587(10)$ & $0.01644(26)$ & $0.01799(78)$ \\
\hline & \multirow[t]{3}{*}{200} & $\delta$ & $2.5071(43)$ & $2.4823(79)$ & $2.457(16)$ & $2.4439(42)$ & $2.4325(78)$ & $2.418(17)$ \\
\hline & & $\mathrm{D}$ & $0.12145(92)$ & $0.1272(19)$ & $0.1338(43)$ & $0.1397(10)$ & $0.1427(21)$ & $0.1471(49)$ \\
\hline & & $f_{\infty}$ & $0.01818(11)$ & $0.01905(28)$ & $0.02036(79)$ & $0.01578(10)$ & $0.01613(25)$ & $0.01685(72)$ \\
\hline \multirow[t]{6}{*}{8000} & \multirow[t]{3}{*}{200} & $\delta$ & $2.4953(35)$ & $2.4839(67)$ & $2.447(14)$ & $2.4320(35)$ & $2.4341(65)$ & $2.408(14)$ \\
\hline & & $\mathrm{D}$ & $0.12364(78)$ & $0.1263(16)$ & $0.1358(36)$ & $0.14230(88)$ & $0.1418(17)$ & $0.1493(42)$ \\
\hline & & $f_{\infty}$ & $0.018323(94)$ & $0.01872(23)$ & $0.02048(67)$ & $0.015919(86)$ & $0.01585(20)$ & $0.01696(61)$ \\
\hline & \multirow[t]{3}{*}{800} & $\delta$ & $2.5003(36)$ & $2.4964(68)$ & $2.484(14)$ & $2.4369(35)$ & $2.4462(66)$ & $2.444(14)$ \\
\hline & & $\mathrm{D}$ & $0.12258(78)$ & $0.1235(16)$ & $0.1266(34)$ & $0.14108(88)$ & $0.1386(17)$ & $0.1394(37)$ \\
\hline & & $f_{\infty}$ & $0.018205(94)$ & $0.01833(23)$ & $0.01890(60)$ & $0.015808(87)$ & $0.01551(20)$ & $0.01561(52)$ \\
\hline \multirow[t]{6}{*}{16000} & \multirow[t]{3}{*}{800} & $\delta$ & $2.4913(53)$ & $2.4619(99)$ & $2.409(22)$ & $2.4283(52)$ & $2.4125(97)$ & $2.370(20)$ \\
\hline & & $\mathrm{D}$ & $0.1245(12)$ & $0.1316(24)$ & $0.1461(62)$ & $0.1432(13)$ & $0.1475(27)$ & $0.1605(63)$ \\
\hline & & $f_{\infty}$ & $0.01842(14)$ & $0.01950(36)$ & $0.0222(12)$ & $0.01601(13)$ & $0.01654(32)$ & $0.01847(96)$ \\
\hline & \multirow[t]{3}{*}{1200} & $\delta$ & $2.4935(53)$ & $2.466(10)$ & $2.423(22)$ & $2.4306(52)$ & $2.4169(98)$ & $2.384(20)$ \\
\hline & & $\mathrm{D}$ & $0.1240(12)$ & $0.1305(25)$ & $0.1421(60)$ & $0.1426(13)$ & $0.1464(27)$ & $0.1562(62)$ \\
\hline & & $f_{\infty}$ & $0.01837(14)$ & $0.01936(37)$ & $0.0215(11)$ & $0.01595(13)$ & $0.01641(32)$ & $0.01786(92)$ \\
\hline \multirow[t]{3}{*}{32000} & \multirow[t]{3}{*}{5000} & $\delta$ & $2.4837(87)$ & $2.479(16)$ & $2.366(33)$ & $2.4208(85)$ & $2.429(16)$ & $2.327(32)$ \\
\hline & & $\mathrm{D}$ & $0.1258(19)$ & $0.1268(38)$ & $0.159(10)$ & $0.1446(22)$ & $0.1422(42)$ & $0.174(11)$ \\
\hline & & $f_{\infty}$ & $0.01844(23)$ & $0.01855(55)$ & $0.0246(21)$ & $0.01603(21)$ & $0.01570(49)$ & $0.0205(18)$ \\
\hline
\end{tabular}

Table 5: Results for the fit $g(\rho)=f_{\infty} \exp \left(-D \rho^{\delta}\right)$ for $\rho>\rho_{\min }$. The first three columns refer to $g(\rho)=f_{M C}(\rho)$, the last three columns to $g(\rho)=f_{M C}(\rho) \rho^{-0.255}$. Here $f_{M C}(\rho)$ is the Monte Carlo EEDF. 


\begin{tabular}{|c|c|c||c|c|c|}
\hline$N$ & $N_{s h}$ & & $\rho_{\min }=1$. & $\rho_{\min }=1.5$ & $\rho_{\min }=2$. \\
\hline 500 & 1 & $\sigma$ & $0.25638(80)$ & $0.2468(11)$ & $0.2237(17)$ \\
& & $f_{\infty}$ & $0.015976(11)$ & $0.016130(17)$ & $0.016540(29)$ \\
& 10 & $\sigma$ & $0.25607(81)$ & $0.2463(11)$ & $0.2230(17)$ \\
& & $f_{\infty}$ & $0.015976(11)$ & $0.016132(17)$ & $0.016548(29)$ \\
\hline 1000 & 1 & $\sigma$ & $0.25146(64)$ & $0.24592(89)$ & $0.2304(14)$ \\
& & $f_{\infty}$ & $0.016019(9)$ & $0.016108(13)$ & $0.016382(23)$ \\
& 20 & $\sigma$ & $0.25030(65)$ & $0.24418(90)$ & $0.2276(14)$ \\
& & $f_{\infty}$ & $0.016027(9)$ & $0.016126(14)$ & $0.016420(23)$ \\
\hline 2000 & 40 & $\sigma$ & $0.24691(61)$ & $0.24285(85)$ & $0.2324(13)$ \\
& & $f_{\infty}$ & $0.016062(9)$ & $0.016128(13)$ & $0.016311(22)$ \\
& 80 & $\sigma$ & $0.24688(63)$ & $0.24276(87)$ & $0.2325(13)$ \\
& & $f_{\infty}$ & $0.016062(9)$ & $0.016129(13)$ & $0.016310(22)$ \\
\hline 4000 & 100 & $\sigma$ & $0.24530(59)$ & $0.24121(82)$ & $0.2351(13)$ \\
& & $f_{\infty}$ & $0.016074(8)$ & $0.016141(12)$ & $0.016247(21)$ \\
& 200 & $\sigma$ & $0.24532(60)$ & $0.24115(84)$ & $0.2350(13)$ \\
& & $f_{\infty}$ & $0.016073(8)$ & $0.016141(13)$ & $0.016248(22)$ \\
\hline 8000 & 200 & $\sigma$ & $0.24274(50)$ & $0.24240(69)$ & $0.2376(11)$ \\
& & $f_{\infty}$ & $0.016103(7)$ & $0.016109(10)$ & $0.016194(18)$ \\
& 800 & $\sigma$ & $0.24278(53)$ & $0.24238(73)$ & $0.2375(11)$ \\
& & $f_{\infty}$ & $0.016102(7)$ & $0.016109(11)$ & $0.016194(19)$ \\
\hline 16000 & 800 & $\sigma$ & $0.24322(75)$ & $0.2434(11)$ & $0.2419(16)$ \\
& & $f_{\infty}$ & $0.016092(11)$ & $0.016088(16)$ & $0.016116(27)$ \\
& \multirow{2}{*}{1200} & $\sigma$ & $0.24330(77)$ & $0.2434(11)$ & $0.2415(16)$ \\
& & $f_{\infty}$ & $0.016090(11)$ & $0.016088(16)$ & $0.016122(27)$ \\
\hline 32000 & 5000 & $\sigma$ & $0.2413(13)$ & $0.2412(18)$ & $0.2440(28)$ \\
& & $f_{\infty}$ & $0.016115(18)$ & $0.016115(27)$ & $0.016066(47)$ \\
\hline
\end{tabular}

Table 6: Results for the fit $f_{M C}(\rho) \exp \left(D \rho^{\delta}\right)=f_{\infty} \rho^{\sigma}$ for $\rho>\rho_{\min } . f_{M C}(\rho)$ is the Monte Carlo EEDF. $D$ and $\delta$ have been set equal to the theoretical predictions, Eqs. (2.9) and (4.32). Data with $\rho>4.5$ have not been included in the fit. 


\begin{tabular}{|c|c|c||c|c|c|c|}
\hline$N$ & $N_{s h}$ & & $\rho_{\max }=0.8$ & $\rho_{\max }=0.6$ & $\rho_{\max }=0.4$ & $\rho_{\max }=0.2$ \\
\hline \hline 500 & 1 & $\theta$ & $0.2177(53)$ & $0.2680(87)$ & $0.303(19)$ & $0.50(13)$ \\
& & $f_{1}$ & $0.01470(5)$ & $0.01542(11)$ & $0.01610(38)$ & $0.0228(54)$ \\
\hline 1000 & 1 & $\theta$ & $0.1975(41)$ & $0.2453(67)$ & $0.274(14)$ & $0.339(55)$ \\
& & $f_{1}$ & $0.01474(4)$ & $0.01543(9)$ & $0.01600(27)$ & $0.0179(18)$ \\
\hline 2000 & 1 & $\theta$ & $0.1906(39)$ & $0.2315(63)$ & $0.270(13)$ & $0.194(73)$ \\
& & $f_{1}$ & $0.01479(3)$ & $0.01539(8)$ & $0.01615(27)$ & $0.0140(19)$ \\
\hline 4000 & 1 & $\theta$ & $0.1895(37)$ & $0.2258(60)$ & $0.284(13)$ & $0.343(61)$ \\
& & $f_{1}$ & $0.01479(3)$ & $0.01536(8)$ & $0.01654(26)$ & $0.0185(21)$ \\
\hline 8000 & 1 & $\theta$ & $0.1874(31)$ & $0.2275(51)$ & $0.251(11)$ & $0.313(53)$ \\
& & $f_{1}$ & $0.01489(3)$ & $0.01546(7)$ & $0.01593(21)$ & $0.0177(17)$ \\
\hline 16000 & 1 & $\theta$ & $0.1721(46)$ & $0.2000(74)$ & $0.186(15)$ & $0.182(75)$ \\
& & $f_{1}$ & $0.01487(4)$ & $0.01527(10)$ & $0.01497(29)$ & $0.0151(21)$ \\
& \multirow{2}{*}{10} & $\theta$ & $0.1745(46)$ & $0.2035(75)$ & $0.191(16)$ & $0.189(76)$ \\
& & $f_{1}$ & $0.01486(4)$ & $0.01528(10)$ & $0.01500(29)$ & $0.0152(21)$ \\
& \multirow{2}{*}{20} & $\theta$ & $0.1750(46)$ & $0.2045(75)$ & $0.194(15)$ & $0.199(77)$ \\
& & $f_{1}$ & $0.01486(4)$ & $0.01529(10)$ & $0.01506(29)$ & $0.0154(22)$ \\
\hline 32000 & 1 & $\theta$ & $0.1416(75)$ & $0.178(12)$ & $0.212(26)$ & $0.28(13)$ \\
& & $f_{1}$ & $0.01482(7)$ & $0.01535(16)$ & $0.01609(51)$ & $0.0179(42)$ \\
& \multirow{2}{*}{10} & $\theta$ & $0.1579(75)$ & $0.200(12)$ & $0.243(26)$ & $0.36(13)$ \\
& & $f_{1}$ & $0.01480(7)$ & $0.01542(16)$ & $0.01637(52)$ & $0.0198(47)$ \\
& \multirow{2}{*}{20} & $\theta$ & $0.1597(75)$ & $0.203(12)$ & $0.247(26)$ & $0.38(13)$ \\
& & $f_{1}$ & $0.01480(7)$ & $0.01543(16)$ & $0.01640(52)$ & $0.0203(48)$ \\
\hline
\end{tabular}

Table 7: Results for the fit $f_{M C}(\rho)=f_{1} \rho^{\theta}$ for $\rho<\rho_{\max } \cdot f_{M C}(\rho)$ is the Monte Carlo EEDF. 